\title{
Electron and muon $g-2$ anomalies in general flavor conserving two-Higgs-doublet models
}

\author{
Francisco J. Botella $\odot^{*}$ and Fernando Cornet-Gomez $\odot^{\dagger}$ \\ Departament de Física Teòrica and IFIC, Universitat de València-CSIC, E-46100, Burjassot, Spain \\ Miguel Nebot $\oplus^{*}$ \\ Departamento de Física and Centro de Física Teórica de Partículas (CFTP), \\ Instituto Superior Técnico (IST), U. de Lisboa (UL), Av. Rovisco Pais 1, P-1049-001 Lisboa, Portugal
}

(Received 13 June 2020; accepted 6 August 2020; published 24 August 2020)

\begin{abstract}
In general two-Higgs-doublet models (2HDMs) without scalar flavor changing neutral couplings (SFCNC) in the lepton sector, the electron, muon, and tau interactions can be decoupled in a robust framework, stable under renormalization group evolution. In this framework, the breaking of lepton flavor universality (LFU) goes beyond the mass proportionality, opening the possibility to accommodate in a simple manner a different behavior among charged leptons. We analyze simultaneously the electron and muon $(g-2)$ anomalies in the context of these general flavor conserving models in the leptonic sector $(\mathrm{g} \ell \mathrm{FC})$. We consider two different models, I-g $\ell \mathrm{FC}$ and II-g $\ell \mathrm{FC}$, in which the quark Yukawa couplings coincide, respectively, with the ones in type I and in type II 2 HDMs. We find two types of solutions that fully reproduce both $(g-2)$ anomalies, and which are compatible with experimental constraints from LEP and LHC, from LFU, from flavor and electroweak physics, and with theoretical constraints in the scalar sector. In the first type of solution, all the new scalars have masses in the $1-2.5 \mathrm{TeV}$ range, the vacuum expectation values (vevs) of both doublets are quite similar in magnitude, and both anomalies are dominated by two loop Barr-Zee contributions. This solution appears in both models. There is a second type of solution, where one loop contributions are dominant in the muon anomaly, all new scalars have masses below $1 \mathrm{TeV}$, and the ratio of vevs is in the range 10-100. The second neutral scalar $\mathrm{H}$ is the lighter among the new scalars, with a mass in the $210-390 \mathrm{GeV}$ range while the pseudoscalar A is the heavier, with a mass in the range 400-900 GeV. The new charged scalar $\mathrm{H}^{ \pm}$is almost degenerate either with the scalar or with the pseudoscalar. This second type of solution only appears in the I-g $\ell$ FC model. Both solutions require the soft breaking of the $\mathbb{Z}_{2}$ symmetry of the Higgs potential.
\end{abstract}

DOI: 10.1103/PhysRevD.102.035023

\section{INTRODUCTION}

After an improved determination of the fine structure constant [1], a new anomaly has emerged [2] concerning the anomalous magnetic moment of the electron $a_{e}=\left(g_{e}-2\right) / 2$ : there is a discrepancy among the experimental determination and the Standard Model (SM) prediction [3-8],

$$
\delta a_{e} \equiv a_{e}^{\mathrm{Exp}}-a_{e}^{\mathrm{SM}}=-(8.7 \pm 3.6) \times 10^{-13}
$$

\footnotetext{
*Francisco.J.Botella@uv.es

${ }^{\dagger}$ Fernando.Cornet@ific.uv.es

*miguel.r.nebot.gomez@tecnico.ulisboa.pt
}

Published by the American Physical Society under the terms of the Creative Commons Attribution 4.0 International license. Further distribution of this work must maintain attribution to the author(s) and the published article's title, journal citation, and DOI. Funded by SCOAP ${ }^{3}$.
Another well known and long standing anomaly concerns the anomalous magnetic moment of the muon [9-15],

$$
\delta a_{\mu} \equiv a_{\mu}^{\mathrm{Exp}}-a_{\mu}^{\mathrm{SM}}=(2.7 \pm 0.9) \times 10^{-9} .
$$

It is to be noticed that the anomalies in Eqs. (1) and (2) have the opposite sign.

Because of this difference of sign, several New Physics solutions addressing Eq. (2) tend to be eliminated as solutions to both Eqs. (2) and (1). In particular, many popular models in which the anomaly scales with the square of the lepton mass [16] tend to generate too large $\delta a_{e}$ with the wrong sign. Some authors [17] argue that if the origin of both anomalies is beyond the SM, the corresponding model must incorporate some sort of effective decoupling between $\mu$ and $e$. Recent beyond-SM explanations of both anomalies can be found in [18-32]. A minimal extension of the SM is the two-Higgs-doublets model (2HDM) [33] which introduces, in general, a new set 
of flavor structures in the Yukawa sector. Those structures could implement the decoupling between $\mu$ and $e$ required to explain $\delta a_{\mu}$ and $\delta a_{e}$. Of course, the most popular 2HDMs shaped by symmetries [34,35], the so-called 2HDMs of types I, II, X, and Y [36-38], do not implement in a straightforward way this decoupling between $\mu$ and $e$, since the new Yukawa couplings in the lepton sector are proportional to the charged lepton mass matrix.

Going one step further in generality, the so-called "Aligned" 2HDM (A2HDM) [39] gives up stability of the model under the renormalization group evolution (RGE) [40] (the model is not shaped by a symmetry). The A2HDM cannot, however, incorporate some effective decoupling between $\mu$ and $e$ since the new Yukawa structures are still proportional to the fermion mass matrices. It is nevertheless interesting to note that the lepton sector of the A2HDM is stable under one loop RGE ${ }^{1}$ [41-43]: scalar flavor changing neutral couplings (SFCNC), absent at tree level, do not appear at one loop.

A generalization of the A2HDM is the general flavor conserving ( $\mathrm{gFC}$ ) 2HDM where, at tree level, all Yukawa couplings are diagonal in the fermion mass basis [44-46]. As in the A2HDM, it has been shown that the charged lepton sector of the gFC-2HDM is one loop stable under RGE, in the sense that SFCNC, absent at tree level, are not generated at one loop [45]. This implies that a well behaved and minimal $2 \mathrm{HDM}$ that can implement the effective decoupling among $\mu$ and $e$ is a gFC-2HDM in the leptonic sector. Since this is all that is required to address the two anomalies in Eqs. (1)-(2), we consider two minimal models in which the quark sector is a $2 \mathrm{HDM}$ of either type I or type II, while the lepton sector corresponds to a gFC-2HDM. We refer to them as models I-g $\ell \mathrm{FC}$ and II-g $\ell \mathrm{FC}$, respectively. Note that these models do not have SFCNC at tree level, neither in the quark nor in the lepton sectors. Additionally, the new Yukawa couplings in the lepton sector are independent of the charged lepton mass matrix. In the appropriate limits, model I-g $\ell$ FC can reproduce $2 \mathrm{HDMs}$ of types I and $\mathrm{X}$ while, similarly, model II-g $\ell \mathrm{FC}$ can reproduce $2 \mathrm{HDMs}$ of types II and $\mathrm{Y}$. In this sense model I-g $\ell \mathrm{FC}$ is a generalization of $2 \mathrm{HDMs}$ of types I and $\mathrm{X}$, while model II-g $\ell \mathrm{FC}$ is instead a generalization of $2 \mathrm{HDMs}$ of types II and $\mathrm{Y}$. The convenience of adopting this kind of generalized flavor conserving $2 \mathrm{HDMs}$ for phenomenological analyses was advocated in [45].

The paper is organized as follows. In Sec. II the models are presented in detail. In Sec. III, the one and two loop contributions to $a_{\ell}$ are revisited. In a simplified analysis it is shown that, with dominating two loop contributions, a new simple scaling law follows:

$$
\frac{\delta a_{e}}{\delta a_{\mu}}=\frac{m_{e} \operatorname{Re}\left(n_{e}\right)}{m_{\mu} \operatorname{Re}\left(n_{\mu}\right)},
$$

\footnotetext{
${ }^{1}$ As in the $\mathrm{SM}$, one is assuming massless neutrinos.
}

with $n_{e}, n_{\mu}$, the new Yukawa couplings of the charged leptons, in the lepton mass basis. In order to solve the discrepancies in Eqs. (1)-(2) through the two loop contributions, the scaling in Eq. (3) requires

$$
\operatorname{Re}\left(n_{\mu}\right)=-\left(15.11_{-7.56}^{+15.11}\right) \operatorname{Re}\left(n_{e}\right)
$$

in the framework of models I-g $\ell \mathrm{FC}$ and II-g $\ell \mathrm{FC}$. Besides solutions with dominating two loop contributions, an additional possibility with relevant one loop contributions is also analyzed (similarly to [2]). In Sec. IV, a number of constraints, relevant for a full analysis, are addressed in detail. In Sec. V, the main results of such a full analysis are presented and discussed. Details concerning some aspects of the different sections are relegated to the Appendices.

\section{THE I-G $\ell$ FC AND II-G $\ell$ FC MODELS}

In 2HDMs, the Yukawa sector of the SM is extended to

$$
\begin{aligned}
\mathcal{L}_{\mathrm{Y}}= & -\bar{Q}_{L}^{0}\left(\Phi_{1} Y_{d 1}+\Phi_{2} Y_{d 2}\right) d_{R}^{0}-\bar{Q}_{L}^{0}\left(\tilde{\Phi}_{1} Y_{u 1}+\tilde{\Phi}_{2} Y_{u 2}\right) u_{R}^{0} \\
& -\bar{L}_{L}^{0}\left(\Phi_{1} Y_{\ell 1}+\Phi_{2} Y_{\ell 2}\right) \ell_{R}^{0}+\text { H.c. },
\end{aligned}
$$

where $\tilde{\Phi}_{j}=i \sigma_{2} \Phi_{j}^{*}$, and, as in the $\mathrm{SM}$, neutrinos are massless (in the leptonic sector only two flavor structures are present). The vacuum expectation values $v_{j}$ of the scalar fields $\Phi_{j}$ are in general nonvanishing; expanding around the vacuum appropriate for electroweak symmetry breaking,

$$
\Phi_{j}=e^{i \theta_{j}}\left(\begin{array}{c}
\varphi_{j}^{+} \\
\frac{v_{j}+\rho_{j}+i \eta_{j}}{\sqrt{2}}
\end{array}\right) .
$$

The so-called Higgs basis [47-49] is defined by

$$
\begin{aligned}
\left(\begin{array}{l}
H_{1} \\
H_{2}
\end{array}\right) & =\mathcal{R}_{\beta}\left(\begin{array}{c}
e^{-i \theta_{1}} \Phi_{1} \\
e^{-i \theta_{2}} \Phi_{2}
\end{array}\right), \quad \text { with } \quad \mathcal{R}_{\beta}=\left(\begin{array}{cc}
c_{\beta} & s_{\beta} \\
-s_{\beta} & c_{\beta}
\end{array}\right), \\
\mathcal{R}_{\beta}^{T} & =\mathcal{R}_{\beta}^{-1},
\end{aligned}
$$

in such a way that only one of the scalar doublets has a nonvanishing vacuum expectation value: $\left\langle H_{1}\right\rangle=\frac{v}{\sqrt{2}}\left(\begin{array}{l}0 \\ 1\end{array}\right)$, $\left\langle H_{2}\right\rangle=\left(\begin{array}{l}0 \\ 0\end{array}\right)$. In Eq. (7), we have used $c_{\beta} \equiv \cos \beta=v_{1} / v$, $s_{\beta} \equiv \sin \beta=v_{2} / v$, with $v_{2}=v_{1}^{2}+v_{2}^{2}=\frac{1}{\sqrt{2} G_{F}}$. Expanding around the vacuum

$$
H_{1}=\left(\begin{array}{c}
G^{+} \\
\frac{v+H^{0}+i G^{0}}{\sqrt{2}}
\end{array}\right), \quad H_{2}=\left(\begin{array}{c}
\mathrm{H}^{+} \\
\frac{R^{0}+i I^{0}}{\sqrt{2}}
\end{array}\right),
$$

the would-be Goldstone bosons $G^{0}, G^{ \pm}$and the physical charged scalar $H^{ \pm}$are already identified. The neutral scalars $\left\{H^{0}, R^{0}, I^{0}\right\}$ are not, in general, the mass eigenstates. 
It is in the Higgs basis where the Yukawa couplings have the simplest interpretation:

$$
\begin{aligned}
\mathcal{L}_{\mathrm{Y}}= & -\frac{\sqrt{2}}{v} \bar{Q}_{L}^{0}\left(H_{1} \mathrm{M}_{d}^{0}+H_{2} \mathrm{~N}_{d}^{0}\right) d_{R}^{0} \\
& -\frac{\sqrt{2}}{v} \bar{Q}_{L}^{0}\left(\tilde{H}_{1} \mathrm{M}_{u}^{0}+\tilde{H}_{2} \mathrm{~N}_{u}^{0}\right) u_{R}^{0} \\
& -\frac{\sqrt{2}}{v} \bar{L}_{L}^{0}\left(H_{1} \mathrm{M}_{\ell}^{0}+H_{2} \mathrm{~N}_{\ell}^{0}\right) \ell_{R}^{0}+\text { H.c. }
\end{aligned}
$$

Since only the neutral component of $H_{1}$ has a nonvanishing vacuum expectation value, the Yukawa couplings $\mathbf{M}_{f}^{0}$, for all the fermions $f=u, d, \ell$, will be the corresponding mass matrices. Going directly to the fermion mass bases, we obtain the relevant new Yukawa structures

$$
\begin{aligned}
\mathcal{L}_{\mathrm{Y}}= & -\frac{\sqrt{2}}{v} \bar{Q}_{L}\left(H_{1} \mathrm{M}_{d}+H_{2} \mathrm{~N}_{d}\right) d_{R} \\
& -\frac{\sqrt{2}}{v} \bar{Q}_{L}\left(\tilde{H}_{1} \mathrm{M}_{u}+\tilde{H}_{2} \mathrm{~N}_{u}\right) u_{R} \\
& -\frac{\sqrt{2}}{v} \bar{L}_{L}\left(H_{1} \mathrm{M}_{\ell}+H_{2} \mathrm{~N}_{\ell}\right) \ell_{R}+\text { H.c. }
\end{aligned}
$$

where $\mathbf{M}_{f}$ are the diagonal fermion mass matrices for $f=$ $u, d, \ell$ and $\mathrm{N}_{f}$ are the new flavor structures that may be able to explain the electron and muon anomalies in Eqs. (1)-(2). As motivated previously, we consider two models.

(i) Model I-g $\ell \mathrm{FC}$ is defined $\mathrm{by}^{2}$

$$
\begin{aligned}
& \mathrm{N}_{u}=t_{\beta}^{-1} \mathrm{M}_{u}, \quad \mathrm{~N}_{d}=t_{\beta}^{-1} \mathrm{M}_{d}, \\
& \mathrm{~N}_{\ell}=\operatorname{diag}\left(n_{e}, n_{\mu}, n_{\tau}\right) .
\end{aligned}
$$

The couplings $\mathrm{N}_{u}, \mathrm{~N}_{d}$ are the same as in $2 \mathrm{HDMs}$ of types I or X.

(ii) Model II-g $\ell \mathrm{FC}$ is defined by

$$
\begin{aligned}
& \mathrm{N}_{u}=t_{\beta}^{-1} \mathbf{M}_{u}, \quad \mathrm{~N}_{d}=-t_{\beta} \mathbf{M}_{d}, \\
& \mathrm{~N}_{\ell}=\operatorname{diag}\left(n_{e}, n_{\mu}, n_{\tau}\right) .
\end{aligned}
$$

The couplings $\mathrm{N}_{u}, \mathrm{~N}_{d}$ are the same as in 2HDMs of types II or Y.

In both models $\mathrm{N}_{\ell}$ is diagonal, arbitrary, and stable at one loop level under RGE, in the sense that it remains diagonal. Note that the effective decoupling among the new couplings of $e$ and $\mu$ that is required in order to explain the $g-2$ anomalies is simply obtained from the independence of $n_{e}$ and $n_{\mu}$.

To complete the definition of the model, in accordance with the fact that the quark sector is a type I or type II $2 \mathrm{HDM}$, we adopt a $\mathbb{Z}_{2}$ symmetric scalar potential

\footnotetext{
${ }^{2}$ Here and in the following, $t_{\beta} \equiv \tan \beta$ and $t_{\beta}^{-1} \equiv \cot \beta$.
}

$$
\begin{aligned}
\mathcal{V}\left(\Phi_{1}, \Phi_{2}\right)= & \mu_{11}^{2} \Phi_{1}^{\dagger} \Phi_{1}+\mu_{22}^{2} \Phi_{2}^{\dagger} \Phi_{2}+\left(\mu_{12}^{2} \Phi_{1}^{\dagger} \Phi_{2}+\text { H.c. }\right) \\
& +\lambda_{1}\left(\Phi_{1}^{\dagger} \Phi_{1}\right)^{2}+\lambda_{2}\left(\Phi_{2}^{\dagger} \Phi_{2}\right)^{2} \\
& +2 \lambda_{3}\left(\Phi_{1}^{\dagger} \Phi_{1}\right)\left(\Phi_{2}^{\dagger} \Phi_{2}\right)+2 \lambda_{4}\left(\Phi_{1}^{\dagger} \Phi_{2}\right)\left(\Phi_{2}^{\dagger} \Phi_{1}\right) \\
& +\left(\lambda_{5}\left(\Phi_{1}^{\dagger} \Phi_{2}\right)^{2}+\text { H.c. }\right) .
\end{aligned}
$$

For $\mu_{12}^{2} \neq 0$, the $\mathbb{Z}_{2}$ symmetry is softly broken. This potential generates the mass matrix of the neutral scalars $\mathcal{M}_{0}^{2}$, which is diagonalized by a $3 \times 3$ real orthogonal matrix $\mathcal{R}$

$$
\mathcal{R}^{T} \mathcal{M}_{0}^{2} \mathcal{R}=\operatorname{diag}\left(m_{\mathrm{h}}^{2}, m_{\mathrm{H}}^{2}, m_{\mathrm{A}}^{2}\right), \quad \mathcal{R}^{-1}=\mathcal{R}^{T} .
$$

The physical neutral scalars $\{\mathrm{h}, \mathrm{H}, \mathrm{A}\}$ are:

$$
\left(\begin{array}{c}
\mathrm{h} \\
\mathrm{H} \\
\mathrm{A}
\end{array}\right)=\mathcal{R}^{T}\left(\begin{array}{c}
H^{0} \\
R^{0} \\
I^{0}
\end{array}\right) .
$$

The Yukawa couplings of the neutral scalars are flavor conserving ${ }^{3}$ :

$$
\mathcal{L}_{N}=-\sum_{S=\mathrm{h}, \mathrm{H}, \mathrm{A}} \sum_{f=u, d, \ell} \sum_{j=1}^{3} \frac{m_{f_{j}}}{v} S \bar{f}_{j}\left(a_{f_{j}}^{S}+i b_{f_{j}}^{S} \gamma_{5}\right) f_{j} .
$$

In the following we focus on a simplified case: we assume that (i) there is no $C P$ violation in the scalar sector and (ii) the new Yukawa couplings are real, $\operatorname{Im}\left(n_{\ell}\right)=0$. In the scalar sector, this corresponds to

$$
\mathcal{R}=\left(\begin{array}{ccc}
s_{\alpha \beta} & -c_{\alpha \beta} & 0 \\
c_{\alpha \beta} & s_{\alpha \beta} & 0 \\
0 & 0 & 1
\end{array}\right) \text {, }
$$

with $s_{\alpha \beta} \equiv \sin (\alpha-\beta)$ and $c_{\alpha \beta} \equiv \cos (\alpha-\beta)$, where $\alpha-\frac{\pi}{2}$ is the mixing angle parametrizing the change of basis from the fields in Eq. (6) to the mass eigenstates in Eq. (15). The alignment limit, in which $\mathrm{h}$ has the same couplings of the SM Higgs, corresponds to $s_{\alpha \beta} \rightarrow 1$. Table I collects the Yukawa couplings, as expressed in Eq. (16), in both models $\mathrm{I}-\mathrm{g} \ell \mathrm{FC}$ and II-g $\ell \mathrm{FC}$. The absence of $C P$ violation is clear from the exact relation $a_{f}^{S} b_{f}^{S}=0$ [50]; one important consequence of this simplification is the absence of new contributions generating electric dipole moments (EDMs), in particular contributions to the electron $\mathrm{EDM} d_{e}$, which is quite constrained: $\left|d_{e}\right|<1.1 \times 10^{-29} \mathrm{e} \cdot \mathrm{cm}[51,52]$. The Yukawa couplings of $\mathrm{H}^{ \pm}$are of the form

\footnotetext{
${ }^{3}$ The general form of the Yukawa couplings is given, for completeness, in Appendix A.
} 
TABLE I. Fermion couplings to neutral scalars.

\begin{tabular}{lccccccc}
\hline \hline & & $a_{u}^{S}$ & $b_{u}^{S}$ & $a_{d}^{S}$ & $b_{d}^{S}$ & $a_{\ell}^{S}$ & $b_{\ell}^{S}$ \\
\hline $\mathrm{I}-\mathrm{g} \ell \mathrm{FC}$ & $\mathrm{h}$ & $s_{\alpha \beta}+c_{\alpha \beta} t_{\beta}^{-1}$ & 0 & $s_{\alpha \beta}+c_{\alpha \beta} t_{\beta}^{-1}$ & 0 & $s_{\alpha \beta}+c_{\alpha \beta} \frac{\operatorname{Re}\left(n_{\ell}\right)}{m_{\ell}}$ & 0 \\
& $\mathrm{H}$ & $-c_{\alpha \beta}+s_{\alpha \beta} t_{\beta}^{-1}$ & 0 & $-c_{\alpha \beta}+s_{\alpha \beta} t_{\beta}^{-1}$ & 0 & $-c_{\alpha \beta}+s_{\alpha \beta} \frac{\operatorname{Re}\left(n_{\ell}\right)}{m_{\ell}}$ & 0 \\
& $\mathrm{~A}$ & 0 & $-t_{\beta}^{-1}$ & 0 & $+t_{\beta}^{-1}$ & 0 & $\frac{\operatorname{Re}\left(n_{\ell}\right)}{m_{\ell}}$ \\
$\mathrm{II}-\mathrm{g} \ell \mathrm{FC}$ & $\mathrm{h}$ & $s_{\alpha \beta}+c_{\alpha \beta} t_{\beta}^{-1}$ & 0 & $s_{\alpha \beta}-c_{\alpha \beta} t_{\beta}$ & 0 & $s_{\alpha \beta}+c_{\alpha \beta} \frac{\operatorname{Re}\left(n_{\ell}\right)}{m_{\ell}}$ & 0 \\
& $\mathrm{H}$ & $-c_{\alpha \beta}+s_{\alpha \beta} t_{\beta}^{-1}$ & 0 & $-c_{\alpha \beta}-s_{\alpha \beta} t_{\beta}$ & 0 & $-c_{\alpha \beta}+s_{\alpha \beta} \frac{\operatorname{Re}\left(n_{\ell}\right)}{m_{\ell}}$ & 0 \\
& $\mathrm{~A}$ & 0 & $-t_{\beta}^{-1}$ & 0 & $-t_{\beta}$ & 0 & $\frac{\operatorname{Re}\left(n_{\ell}\right)}{m_{\ell}}$ \\
\hline \hline
\end{tabular}

TABLE II. Fermion couplings to $\mathrm{H}^{ \pm}$.

\begin{tabular}{lcccc}
\hline \hline & $\alpha_{i j}^{q}$ & $\beta_{i j}^{q}$ & $\alpha_{i j}^{l}$ & $\beta_{i j}^{l}$ \\
\hline $\mathrm{I}-\mathrm{g} \ell \mathrm{FC}$ & $V_{j i}^{*} t_{\beta}^{-1}\left(m_{u_{j}}-m_{d_{i}}\right)$ & $V_{j i}^{*} t_{\beta}^{-1}\left(m_{u_{j}}+m_{d_{i}}\right)$ & $-\operatorname{Re}\left(n_{\ell_{i}}\right) \delta_{i j}$ & $\operatorname{Re}\left(n_{\ell_{i}}\right) \delta_{i j}$ \\
$\mathrm{II}-\mathrm{g} \ell \mathrm{FC}$ & $V_{j i}^{*}\left(t_{\beta}^{-1} m_{u_{j}}+t_{\beta} m_{d_{i}}\right)$ & $V_{j i}^{*}\left(t_{\beta}^{-1} m_{u_{j}}-t_{\beta} m_{d_{i}}\right)$ & $-\operatorname{Re}\left(n_{\ell_{i}}\right) \delta_{i j}$ & $\operatorname{Re}\left(n_{\ell_{i}}\right) \delta_{i j}$ \\
\hline \hline
\end{tabular}

$$
\begin{aligned}
\mathcal{L}_{C h}= & -\frac{1}{\sqrt{2} v} \sum_{f=q, l} \sum_{j, k=1}^{3}\left\{\mathrm{H}^{-} \bar{f}_{-\frac{1}{2}, j}\left(\alpha_{j k}^{f}+i \beta_{j k}^{f} \gamma_{5}\right) f_{\frac{1}{2}, k}\right. \\
& \left.+\mathrm{H}^{+} \bar{f}_{\frac{1}{2}, k}\left(\alpha_{j k}^{f *}+i \beta_{j k}^{f *} \gamma_{5}\right) f_{-\frac{1}{2}, j}\right\},
\end{aligned}
$$

where $q_{+\frac{1}{2}, j}=u_{j}, q_{-\frac{1}{2}, j}=d_{j}, l_{+\frac{1}{2}, j}=\nu_{j}, l_{-\frac{1}{2}, j}=\ell_{j}$, and the corresponding couplings are given in Table II. Note that the Yukawa couplings of the charged leptons in Tables I and II are the same in both models I-g $\ell \mathrm{FC}$ and II-g $\ell \mathrm{FC}$.

\section{THE NEW CONTRIBUTIONS TO $\delta a_{\ell}$}

The full prediction $a_{\ell}^{\mathrm{Th}}$ of the anomalous magnetic moments of $\ell=e, \mu$ has the form

$$
a_{\ell}^{\mathrm{Th}}=a_{\ell}^{\mathrm{SM}}+\delta a_{\ell},
$$

with $a_{\ell}^{\mathrm{SM}}$ the SM contribution and $\delta a_{\ell}$ the corrections due to the model. To solve the discrepancies in Eqs. (1)-(2), the aim is to obtain $\delta a_{e} \simeq \delta a_{e}^{\text {Exp }}$ and $\delta a_{\mu} \simeq \delta a_{\mu}^{\text {Exp }}$ within models I-g $\ell \mathrm{FC}$ and II-gII-g $\ell \mathrm{FC}$. It is convenient to introduce $\Delta_{\ell}$ following:

$$
\delta a_{\ell}=K_{\ell} \Delta_{\ell}, \quad K_{\ell}=\frac{1}{8 \pi^{2}}\left(\frac{m_{\ell}}{v}\right)^{2}=\frac{1}{8 \pi^{2}}\left(\frac{g m_{\ell}}{2 M_{W}}\right)^{2} .
$$

The quantities $K_{\ell}$ collect the typical factors arising in one loop contributions; since $K_{e} \simeq 5.5 \times 10^{-14}$ and $K_{\mu} \simeq 2.3 \times 10^{-9}$, in order to reproduce the anomalies we roughly need

$$
\Delta_{e} \simeq-16, \quad \Delta_{\mu} \simeq 1 .
$$

It is well known that in the type of models considered here, both one loop [53] or two loop Barr-Zee contributions [54-59] can be dominant. Complete expressions used in the full analyses of Sec. V, can be found in Appendix B. For the moment, we consider in this section two approximations: we only keep leading terms in a $\left(m_{\ell} / m_{\mathrm{S}}\right)^{2}$ expansion (for the different scalars $\mathrm{S}=\mathrm{h}, \mathrm{H}, \mathrm{A}$ ), and the alignment limit $s_{\alpha \beta} \rightarrow 1$. With these approximations, the one loop contribution to $\Delta_{\ell}$ in Eq. (20) is

$$
\Delta_{\ell}^{(1)} \simeq n_{\ell}^{2}\left(\frac{I_{\ell \mathrm{H}}}{m_{\mathrm{H}}^{2}}-\frac{I_{\ell \mathrm{A}}-2 / 3}{m_{\mathrm{A}}^{2}}-\frac{1}{6 m_{\mathrm{H}^{ \pm}}^{2}}\right),
$$

where

$$
I_{\ell \mathrm{S}}=-\frac{7}{6}-2 \ln \left(\frac{m_{\ell}}{m_{\mathrm{S}}}\right)
$$

Equation (22) applies to both model I-g $\ell \mathrm{FC}$ and II-g $\ell \mathrm{FC}$. We do not consider light scalars or pseudoscalars (see Ref. [29]): in the different analyses it is assumed that $h$ is the lightest scalar, i.e., $m_{\mathrm{h}}<m_{\mathrm{H}}, m_{\mathrm{A}}$. For a typical range $m_{\mathrm{S}} \in[0.2 ; 2.0] \mathrm{TeV}$, the loop functions $I_{\ell \mathrm{S}}$ obey

$$
I_{e \mathrm{~S}} \in[24.6 ; 29.2], \quad I_{\mu \mathrm{S}} \in[13.9 ; 18.5],
$$

and thus the dominant contributions to $\Delta_{\ell}^{(1)}$ in Eq. (22) are the logarithmically enhanced contributions from $\mathrm{H}$ and $\mathrm{A}$. Then, $\Delta_{e} \simeq-16$ can only arise from the negative sign of the A pseudoscalar contribution: $\Delta_{e} \simeq-\left[\operatorname{Re}\left(n_{e}\right)\right]^{2} I_{e \mathrm{~A}} / m_{\mathrm{A}}^{2}$. Taking into account the $I_{e \mathrm{~A}}$ value in Eq. (24), it would 

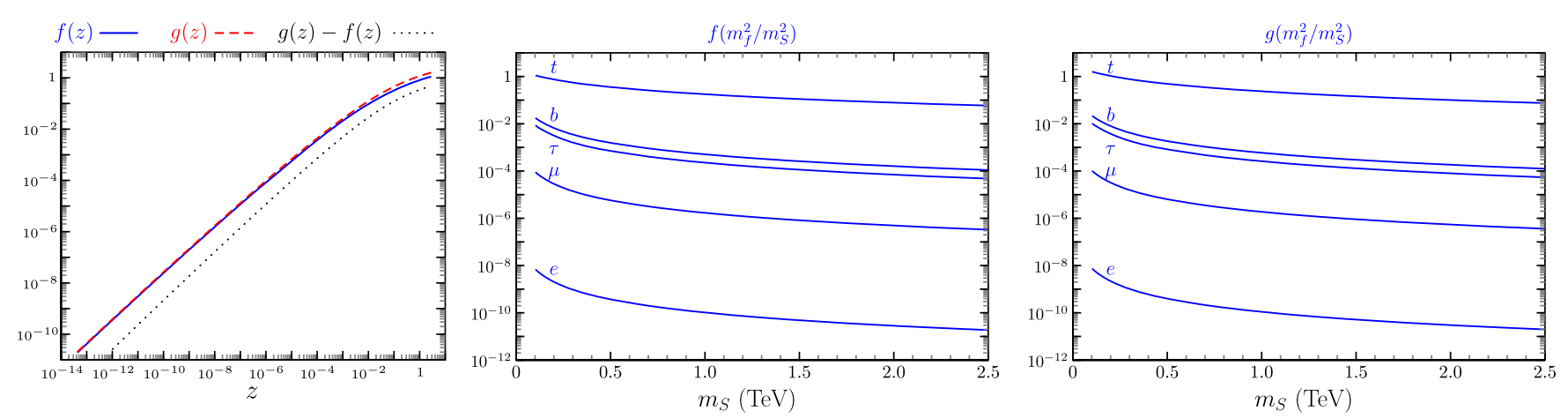

FIG. 1. Loop functions.

require $\left[\operatorname{Re}\left(n_{e}\right)\right]^{2} \sim m_{\mathrm{A}}^{2}$, which can easily violate perturbativity requirements in the Yukawa sector or constraints from resonant dilepton searches. Consequently, we do not expect an explanation of $\delta a_{e}$ in terms of one loop contributions. For $\delta a_{\mu}$, any relevant one loop contribution in Eq. (22) should arise from the $\mathrm{H}$ contribution attending, again, to the required sign and the logarithmically enhanced value of $I_{\mu \mathrm{H}}$ in Eq. (24): $\Delta_{\mu} \simeq\left[\operatorname{Re}\left(n_{\mu}\right)\right]^{2} I_{\mu \mathrm{H}} / m_{\mathrm{H}}^{2}$. For $I_{\mu \mathrm{H}} \simeq 16$ such a contribution needs $\left[\operatorname{Re}\left(n_{\mu}\right)\right]^{2} \sim\left[m_{\mathrm{H}} / 4\right]^{2}$, that is a not too heavy $\mathrm{H}$ (in order to have reasonably perturbative $n_{\mu}$ ) and $m_{\mathrm{A}}>m_{\mathrm{H}}$ in order to avoid cancellations with wrong sign contributions. In the same approximation (leading $m_{\ell} / m_{\mathrm{S}}$ terms and $s_{\alpha \beta} \rightarrow 1$ ), the two loop contributions are dominated by Barr-Zee diagrams in which the internal fermion loop is connected with the external lepton via one virtual photon and one virtual neutral scalar $\mathrm{H}$ or $\mathrm{A}$. The leading contribution to $\Delta_{\ell}$ in Eq. (20) is (for detailed expressions, see Appendix B)

$$
\Delta_{\ell}^{(2)}=-\left(\frac{2 \alpha}{\pi}\right)\left(\frac{n_{\ell}}{m_{\ell}}\right) F
$$

The factor $F$ depends on the masses of the fermions in the closed loop, on the couplings of those fermions to $\mathrm{H}$ and $\mathrm{A}$, and, of course, on $m_{\mathrm{H}}$ and $m_{\mathrm{A}}$; it is consequently different in models $\mathrm{I}-\mathrm{g} \ell \mathrm{FC}$ and $\mathrm{II}-\mathrm{g} \ell \mathrm{FC}$ :

$$
\begin{aligned}
F_{\mathrm{I}}= & \frac{\cot \beta}{3}\left[4\left(f_{t \mathrm{H}}+g_{t \mathrm{~A}}\right)+\left(f_{b \mathrm{H}}-g_{b \mathrm{~A}}\right)\right] \\
& +\frac{\operatorname{Re}\left(n_{\tau}\right)}{m_{\tau}}\left(f_{\tau \mathrm{H}}-g_{\tau \mathrm{A}}\right), \\
F_{\mathrm{II}}= & \frac{\cot \beta}{3}\left[4\left(f_{t \mathrm{H}}+g_{t \mathrm{~A}}\right)-\tan ^{2} \beta\left(f_{b \mathrm{H}}-g_{b \mathrm{~A}}\right)\right] \\
& +\frac{\operatorname{Re}\left(n_{\tau}\right)}{m_{\tau}}\left(f_{\tau \mathrm{H}}-g_{\tau \mathrm{A}}\right),
\end{aligned}
$$

where

$$
f_{f \mathrm{~S}} \equiv f\left(\frac{m_{f}^{2}}{m_{\mathrm{S}}^{2}}\right), \quad g_{f \mathrm{~S}} \equiv g\left(\frac{m_{f}^{2}}{m_{\mathrm{S}}^{2}}\right)
$$

The functions $f(z)$ and $g(z)$ are defined in Appendix B; they are represented in Fig. 1. Their main features are: (i) $f(z) \simeq g(z)$ in the whole range of interest, (ii) the largest values correspond to the heavier fermion (the top quark), (iii) the values of $f$ and $g$ for the top quark contributions vary between 0.1 and 1 in the relevant range of scalar masses. Considering the dominant top quark terms, for $t_{\beta} \simeq$ 1 and $m_{\mathrm{H}} \simeq m_{\mathrm{A}}$, it is easy to realize that for $m_{\mathrm{H}} \sim 1-2 \mathrm{TeV}$, $\delta a_{e}$ can be explained with Yukawa couplings $\operatorname{Re}\left(n_{e}\right) \sim$ 3-7 GeV $\left[\operatorname{Re}\left(n_{e}\right)>0\right.$ gives the right sign of $\left.\delta a_{e}\right]$. If we assume that $\delta a_{\mu}$ must also be explained by the same kind of dominant Barr-Zee two loop contributions, which are independent of the specific charged lepton, it is straightforward that

$$
\delta a_{\mu}=\frac{m_{\mu} \operatorname{Re}\left(n_{\mu}\right)}{m_{e} \operatorname{Re}\left(n_{e}\right)} \delta a_{e} .
$$

With this relation, the origin of the different signs of $\delta a_{e}$ and $\delta a_{\mu}$ relies on the freedom to have $\operatorname{Re}\left(n_{e}\right)$ and $\operatorname{Re}\left(n_{\mu}\right)$ with opposite signs, $\operatorname{Re}\left(n_{\mu}\right) \simeq-15 \operatorname{Re}\left(n_{e}\right)$, as anticipated in Eq. (3). In terms of $\operatorname{Re}\left(n_{\mu}\right)$, with the same assumptions $\left(t_{\beta} \sim 1, m_{\mathrm{A}} \sim m_{\mathrm{H}} \sim 1-2 \mathrm{TeV}\right), \operatorname{Re}\left(n_{\mu}\right) \in-[45 ; 105] \mathrm{GeV}$. The previous arguments apply to both models, I-g $\ell \mathrm{FC}$ and II- $\ell \ell \mathrm{FC}$, since $4\left(f_{t \mathrm{H}}+g_{t \mathrm{~A}}\right)$ is the dominant term in both $F_{\mathrm{I}}$ and $F_{\text {II }}$.

Attending to the flavor constraints discussed in Sec. IV ( $B_{d}$ and $B_{s}$ meson mixings, $b \rightarrow s \gamma$ radiative decays), $t_{\beta} \ll$ 1 are excluded in 2HDMs of types I and II, and thus also in $\mathrm{I}-\mathrm{g} \ell \mathrm{FC}$ and II-g $\ell \mathrm{FC}$ models; there is no need to discuss the $t_{\beta} \ll 1$ regime.

Let us now analyze the two loop Barr-Zee contributions in Eq. (26) for large values of $t_{\beta}$. As a reference, consider the analysis above with $t_{\beta} \sim 1$ and $m_{\mathrm{A}} \sim m_{\mathrm{H}} \sim 1-2 \mathrm{TeV}$; for definiteness we now take $t_{\beta}=50$. For large $t_{\beta}$, it is clear that these contributions in models $\mathrm{I}-\mathrm{g} \ell \mathrm{FC}$ and II-g $\ell \mathrm{FC}$ are quite different. Starting with model $\mathrm{I}-\mathrm{g} \ell \mathrm{FC}$, in order to maintain the right value of $\delta a_{e}$, the $t_{\beta}$ suppression in $\operatorname{Re}\left(n_{e}\right) t_{\beta}^{-1}\left(f_{t \mathrm{H}}+g_{t \mathrm{~A}}\right)$ can be compensated with smaller $m_{\mathrm{H}}, m_{\mathrm{A}}$, and larger $\operatorname{Re}\left(n_{e}\right)$. For example, $m_{\mathrm{A}} \sim m_{\mathrm{H}} \sim$ $200 \mathrm{GeV}$ gives an increase of the loop functions by a 
factor of 10 with respect to $m_{\mathrm{A}} \sim m_{\mathrm{H}} \sim 1-2 \mathrm{TeV}$; increasing then $\operatorname{Re}\left(n_{e}\right)$ by a factor of 5 , the suppression $t_{\beta}^{-1}=$ $1 / 50$ is compensated. Therefore, the discrepancy in $\delta a_{e}$ can be explained in the $\mathrm{I}-\mathrm{g} \ell \mathrm{FC}$ model through two loop contributions, for large values of $t_{\beta}$ and $\operatorname{Re}\left(n_{e}\right) \sim$ $15-35 \mathrm{GeV}$. The question now is if one can explain, with the two loop contributions, the muon anomaly $\delta a_{\mu}$. Attending to Eq. (28), one would need $\operatorname{Re}\left(n_{\mu}\right) \in$ $-[225 ; 505] \mathrm{GeV}$, which would be in conflict with perturbativity requirements in the Yukawa sector. However, as the discussion on one loop contributions after Eq. (22) shows, for light $m_{\mathrm{H}}$, e.g., $m_{\mathrm{H}} \in[200 ; 400] \mathrm{GeV}, \delta a_{\mu}$ can be obtained with $\mathrm{H}$-mediated one loop contributions, and $m_{\mathrm{A}}>m_{\mathrm{H}}$ to avoid cancellations. One needs $\left|\operatorname{Re}\left(n_{\mu}\right)\right| \sim$ $m_{\mathrm{H}} / 4$, in which case $\left|\operatorname{Re}\left(n_{\mu}\right)\right| \in[50 ; 100] \mathrm{GeV}$ is acceptable from the perturbativity point of view.

Summarizing the previous discussion, we envisage, at least, two kinds of solutions:

(i) The first is realized with scalars having masses in the 1-2 TeV range, $t_{\beta} \sim 1$, and both anomalies produced by two loop Barr-Zee contributions. The coupling of electrons to the new scalar and pseudoscalar, $\operatorname{Re}\left(n_{e}\right)$, should be in the few $\mathrm{GeV}$ range. Following Eq. (28), the corresponding muon coupling is larger. This first solution can appear, a priori, in both I-g $\ell$ FC and II-gII-g $\ell$ FC models. In Sec. V we refer to this first type of solution as "solution [A]".

(ii) The second solution corresponds to a lighter $\mathrm{H}$, $m_{\mathrm{H}} \in[200 ; 400] \mathrm{GeV}$ and a heavier A; the required values of $t_{\beta}$ are larger, $t_{\beta} \gg 1$. In this second solution, the electron anomaly is obtained with two loop contributions while the muon anomaly is one loop controlled; contrary to the first solution, there is no linear relation among $\operatorname{Re}\left(n_{\mu}\right)$ and $\operatorname{Re}\left(n_{e}\right)$. This second kind of solution can clearly appear in the $\mathrm{I}-\mathrm{g} \ell \mathrm{FC}$ model, but in this simplified analysis it cannot be elucidated if this possibility is also open in the II- $\mathrm{g} \ell \mathrm{FC}$ model. Anticipating the results of the complete numerical analyses of Sec. V, this will not be the case: within the II-g $\ell \mathrm{FC}$ model there is no solution with large $t_{\beta}$ and relatively light $\mathrm{H}$. In Sec. V we refer to this second type of solution as "solution [B]". Notice also that, a priori, this second kind of solution might be obtained with both signs of $\operatorname{Re}\left(n_{\mu}\right)$.

\section{CONSTRAINTS}

In this section we discuss the different constraints that can play a relevant role in the detailed analyses of Sec. V. Each constraint is implemented as $\chi^{2}$ function; a global $\chi^{2}$ function, the sum of all separate contributions, is used to drive the analyses (for an efficient exploration of parameter space we employ Markov chain Monte Carlo techniques) and represent the relevant regions for different parameters and observables. The usual form $\chi_{\mathcal{O}}^{2}=\left(\frac{\mathcal{O}_{\mathrm{Th}}-\mathcal{O}_{\mathrm{Exp}}}{\sigma_{\mathrm{Exp}}}\right)^{2}$ is adopted for the constraint corresponding to an observable $\mathcal{O}$, where the experimental input is a measurement $\mathcal{O}_{\operatorname{Exp}}$ with uncertainty $\sigma_{\operatorname{Exp}}$ and the theoretical prediction is $\mathcal{O}_{\mathrm{Th}}$ (for correlated measurements or asymmetric uncertainties, appropriate modifications are incorporated). Not all constraints are implemented through the usual $\chi^{2}$ form: it is not adequate to incorporate the bounds for perturbativity requirements of the Yukawa couplings in Sec. IV C and the bounds obtained in LHC searches in Sec. IV G. In those cases, instead of sharp bounds or cuts, the bounds are implemented as described in the respective subsections. Furthermore, since $\delta a_{e}$ and $\delta a_{\mu}$ have a role much more important than the rest of constraints, they are also incorporated in a different manner (as described in detail below) to ensure that the analysis focuses on the ability of the model to reproduce values which are clearly non-SM.

\section{A. $\delta a_{\ell}$ constraints for the numerical analyses}

The main motivation of this work is to accommodate the departures from SM expectations in the anomalous magnetic moments of both electron and muon. We now discuss how these departures are implemented as constraints in the analyses presented in Sec. V. The $g-2$ anomalies $\delta a_{\ell}^{\text {Exp }}=$ $a_{\ell}^{\text {Exp }}-a_{\ell}^{\mathrm{SM}}$ in Eqs. (1)-(2) are

$$
\begin{aligned}
& \delta a_{e}^{\text {Exp }}=-(8.7 \pm 3.6) \times 10^{-13}, \\
& \delta a_{\mu}^{\text {Exp }}=(2.7 \pm 0.9) \times 10^{-9} .
\end{aligned}
$$

The theoretical prediction in the present models is $a_{\ell}^{\mathrm{Th}}=$ $\delta a_{\ell}+a_{\ell}^{\mathrm{SM}}$ and thus a simple and natural measure of their ability to accommodate the experimental results is a $\chi^{2}$ function

$$
\chi_{0}^{2}\left(\delta a_{e}, \delta a_{\mu}\right)=\left(\frac{\delta a_{e}-c_{e}}{\sigma_{e}}\right)^{2}+\left(\frac{\delta a_{\mu}-c_{\mu}}{\sigma_{\mu}}\right)^{2},
$$

where $\delta a_{\ell}^{\mathrm{Exp}}=c_{\ell} \pm \sigma_{\ell}$ in Eq. (29).

The interest in explanations of the experimental results in terms of non-SM contributions is due to the $3-4 \sigma$ deviation $\chi_{0}^{2}(0,0) \simeq 15$. For the numerical exploration of the regions in parameter space which could provide such an explanation, rather than including a contribution $\chi_{0}^{2}\left(\delta a_{e}, \delta a_{\mu}\right)$ in the global $\chi^{2}$, we impose a stronger requirement: instead of $\chi_{0}^{2}\left(\delta a_{e}, \delta a_{\mu}\right)$ we include

$$
\begin{aligned}
& \chi^{2}\left(\delta a_{e}, \delta a_{\mu}\right) \\
& = \begin{cases}0, & \text { if } \chi_{0}^{2}\left(\delta a_{e}, \delta a_{\mu}\right) \leq \frac{1}{4}, \\
10^{6} \times\left(\chi_{0}^{2}\left(\delta a_{e}, \delta a_{\mu}\right)-\frac{1}{4}\right), & \text { if } \chi_{0}^{2}\left(\delta a_{e}, \delta a_{\mu}\right)>\frac{1}{4},\end{cases}
\end{aligned}
$$

in order to guarantee that the models reproduce both anomalies simultaneously within less than $\frac{1}{2} \sigma_{\ell}$ of the 


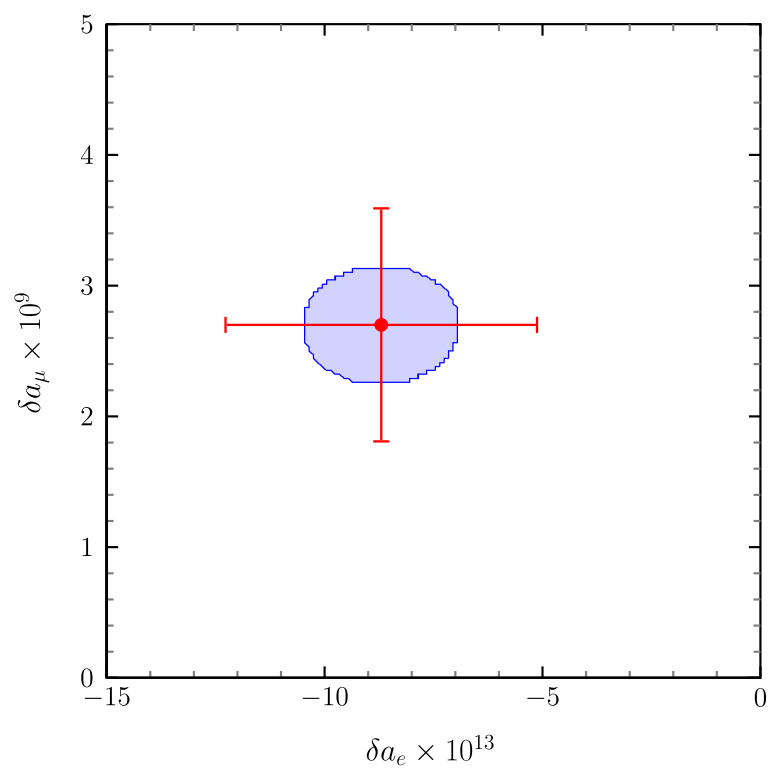

FIG. 2. Allowed $\delta a_{\mu}$ vs $\delta a_{e}$ region.

central values (in the regions of interest, Eq. (31) approximates a "sharp" box function). This approach is adopted in order to ensure that, when representing allowed regions at a given confidence level in the next section, they do not include regions where one or both anomalies are only partially reproduced. For illustration, Fig. 2 shows the allowed region obtained in the complete numerical analyses (which is identical in both models); that is, in the results of Sec. V, within all the represented allowed regions, the values of $\delta a_{e}$ and $\delta a_{\mu}$ belong to the allowed region of Fig. 2. Notice, finally, that the SM prediction $a_{\ell}^{\mathrm{SM}}$ includes Higgs-mediated contributions: since these are just the $\mathrm{h}$ mediated contributions for exact alignment $s_{\alpha \beta}=1$, they have to be subtracted from the New Physics contributions to $\delta a_{\ell}$ mediated by h (quantitatively, however, this subtlety is rather irrelevant).

\section{B. Scalar sector}

For the scalar sector, we use the set of independent parameters $\quad\left\{v, m_{\mathrm{h}}, m_{\mathrm{H}}, m_{\mathrm{A}}, m_{\mathrm{H}^{ \pm}}, t_{\beta}, \alpha-\beta, \mu_{12}^{2}\right\} \quad$ (from which the quartic parameters $\lambda_{j}$ are obtained) with $v=$ $246 \mathrm{GeV}$ and $m_{\mathrm{h}}=125 \mathrm{GeV}$. We require the potential to be bounded from below following [60], we also require the quartic parameters to respect perturbativity and perturbative unitarity in $2 \rightarrow 2$ scattering [61-64] (see also [65-67]), and finally the corrections to the oblique parameters $S$ and $T$, which depend on the scalar masses and $\alpha-\beta$, have to be in agreement with electroweak precision data [68].

\section{Fermion sector}

In the fermion sector, the new couplings $n_{\ell}$ arise from the dimensionless Yukawa couplings $Y_{\ell j}$ in Eq. (5). If one required that Yukawa couplings remain perturbative, for example not exceeding $\mathcal{O}(1)$ values, this would translate into $n_{\ell}$ 's smaller than $v / \sqrt{2} \simeq 174 \mathrm{GeV}$. We adopt a more conservative approach, and include a contribution of the following form to the global $\chi^{2}$ function driving the numerical analyses:

$$
\chi_{\text {Pert }}^{2}\left(n_{\ell}\right)=\left\{\begin{array}{l}
0, \quad \text { for }\left|n_{\ell}\right| \leq n_{0}, \\
\left(\frac{\left|n_{\ell}\right|-n_{0}}{\sigma_{n_{0}}}\right)^{2}, \quad \text { for }\left|n_{\ell}\right|>n_{0} .
\end{array}\right.
$$

We choose $n_{0}=95 \mathrm{GeV}$ and $\sigma_{n_{0}}=1 \mathrm{GeV}$. One could have adopted a crude requirement such as imposing for example $\left|n_{\ell}\right| \leq 100 \mathrm{GeV}$ with a sharp cut: Eq. (32) is simply a smooth version (more convenient for numerical purposes) of that kind of requirement.

\section{Higgs signal strengths}

Concerning the $125 \mathrm{GeV}$ Higgs-like scalar, agreement with the observed production $\times$ decay signal strengths of the usual channels is also imposed [69-72]. The measured signal strengths, with uncertainties reaching the $10 \%$ level, tend to favor the alignment limit in the scalar sector; it is to be noticed that since the models require $\left|\operatorname{Re}\left(n_{e}\right)\right| \gg m_{e}$ and $\left|\operatorname{Re}\left(n_{\mu}\right)\right| \gg m_{\mu}$, the Higgs measurements in the $\mu^{+} \mu^{-}$ channel such as $[73,74]$ are even more effective in forcing that alignment limit. Constraints on the total width $\Gamma_{\mathrm{h}}$, arising from off-shell $(\mathrm{ggF}+\mathrm{VBF}) \rightarrow \mathrm{h}^{(*)} \rightarrow W W^{(*)}$ [75], are also included [76,77], even if in the models considered here their effect is negligible in the alignment limit. For additional details, see $[45,78,79]$.

\section{E. $\mathbf{H}^{ \pm}$mediated contributions}

Flavor transitions mediated by $W^{ \pm}$can receive new contributions where $W^{ \pm} \rightarrow \mathrm{H}^{ \pm}$. For tree level processes involving leptons, one refers to "Lepton Flavor Universality" constraints; we also consider constraints at the loop level in the quark sector.

One may also worry about too large $\mathrm{H}^{ \pm}$-mediated contributions to processes like $\ell_{j} \rightarrow \ell_{k} \gamma$ : since in the present models we are considering massless neutrinos, lepton family numbers are conserved-i.e., there is a $[U(1)]^{3}$ symmetry—and such processes are absent.

\section{Lepton flavor universality}

Contributions mediated by $\mathrm{H}^{ \pm}$modify the leptonic decays $\ell_{j} \rightarrow \ell_{k} \nu \bar{\nu}$ :

$$
\begin{aligned}
\Gamma\left(\ell_{j} \rightarrow \ell_{k} \nu \bar{\nu}\right)= & \frac{G_{F}^{2}}{192 \pi^{3}} m_{\ell_{j}}^{5} \mathrm{f}\left(x_{k j}\right)\left(1+\frac{1}{4}\left|g_{j \rightarrow k}^{S, \mathrm{RR}}\right|^{2}\right. \\
& \left.+2 \operatorname{Re}\left(g_{j \rightarrow k}^{S, \mathrm{RR}}\right) \frac{m_{\ell_{k}}}{m_{\ell_{j}}} \frac{\mathrm{g}\left(x_{k j}\right)}{\mathrm{f}\left(x_{k j}\right)}\right) \times\left(1+\Delta_{\mathrm{RC}}^{\ell_{j} \ell_{k}}\right),
\end{aligned}
$$


where $\mathrm{f}(x)$ and $\mathrm{g}(x)$ are the usual phase space integrals ${ }^{4}$ [80], $\left.x_{k j} \equiv\left(m_{\ell_{k}} / m_{\ell_{j}}\right)^{2}\right)$ and $\Delta_{\mathrm{RC}}^{\ell_{j} \ell_{k}}$ correspond to QED radiative corrections and most importantly,

$$
g_{j \rightarrow k}^{S, \mathrm{RR}}=-\frac{n_{\ell_{j}}^{*} n_{\ell_{k}}}{m_{\mathrm{H}^{ \pm}}^{2}} .
$$

The notation $g_{j \rightarrow k}^{S, R R}$ reflects the fact that in the present models the new contributions only affect, in an effective description, the operator $\bar{\nu}_{L} \ell_{j R} \bar{\ell}_{k R} \nu_{L}$. The ratios

$$
R\left[\frac{\ell_{a} \rightarrow \ell_{b}}{\ell_{\alpha} \rightarrow \ell_{\beta}}\right] \equiv \frac{\Gamma\left(\ell_{a} \rightarrow \ell_{b} \nu \bar{\nu}\right)}{\Gamma\left(\ell_{a} \rightarrow \ell_{b} \nu \bar{\nu}\right)_{\mathrm{SM}}} \frac{\Gamma\left(\ell_{\alpha} \rightarrow \ell_{\beta} \nu \bar{\nu}\right)_{\mathrm{SM}}}{\Gamma\left(\ell_{\alpha} \rightarrow \ell_{\beta} \nu \bar{\nu}\right)}
$$

give the following constraints [81]:

$$
\begin{aligned}
& R\left[\frac{\tau \rightarrow \mu}{\tau \rightarrow e}\right]=1+(3.8 \pm 3.2) \times 10^{-3}, \\
& R\left[\frac{\tau \rightarrow e}{\mu \rightarrow e}\right]=1+(2.4 \pm 2.3) \times 10^{-3}
\end{aligned}
$$

In addition, measurements of decay spectra with polarized leptons impose

$$
\begin{aligned}
& \left|g_{\mu \rightarrow e}^{S, \mathrm{RR}}\right|<0.035 \text { at } 90 \% \mathrm{CL}, \\
& \left|g_{\tau \rightarrow \mu}^{S, \mathrm{RR}}\right|<0.72 \text { at } 95 \% \mathrm{CL}, \quad\left|g_{\tau \rightarrow e}^{S, \mathrm{RR}}\right|<0.7 \text { at } 95 \% \mathrm{CL} .
\end{aligned}
$$

Besides purely leptonic decays $\ell_{j} \rightarrow \ell_{k} \bar{\nu} \nu$, leptonic decay modes like $K, \pi \rightarrow e \nu, \mu \nu$ and $\tau \rightarrow K \nu, \pi \nu$, provide additional constraints on the different $n_{\ell}$ (together with the $t_{\beta}$ dependence of the quark couplings with $\mathrm{H}^{ \pm}$). In particular, we consider ratios

$R_{\ell_{1} \ell_{2}}^{P}=\frac{\Gamma\left(P^{+} \rightarrow \ell_{1}^{+} \nu\right)}{\Gamma\left(P^{+} \rightarrow \ell_{1}^{+} \nu\right)_{\mathrm{SM}}} \frac{\Gamma\left(P^{+} \rightarrow \ell_{2}^{+} \nu\right)_{\mathrm{SM}}}{\Gamma\left(P^{+} \rightarrow \ell_{2}^{+} \nu\right)}=\frac{\left|1-\Delta_{\ell_{1}}^{P}\right|^{2}}{\left|1-\Delta_{\ell_{2}}^{P}\right|^{2}}$,

where the quark content of $P^{+}$is $u_{i} \bar{d}_{j}$, and

$$
\left|1-\Delta_{\ell_{a}}^{P}\right|^{2} \equiv\left|1-\frac{M_{P}^{2}}{M_{H^{ \pm}}^{2}} \frac{k_{u} m_{u_{i}}+k_{d}^{*} m_{d_{j}}}{m_{u_{i}}+m_{d_{j}}} \frac{n_{\ell_{a}}}{m_{\ell_{a}}}\right|^{2},
$$

with $k_{u}=k_{d}=t_{\beta}^{-1}$ in model I-g $\ell \mathrm{FC}$ and $k_{u}=-k_{d}^{-1}=t_{\beta}^{-1}$ in model II-g $\ell$ FC. Notice the enhanced sensitivity of these observables due to the $\frac{n_{\ell_{a}}}{m_{\ell_{a}}}$ factor: unlike the SM amplitude, the new $\mathrm{H}^{ \pm}$-mediated amplitude is not helicity suppressed.

\footnotetext{
${ }^{4} \mathrm{f}(x)=1-8 x+8 x^{3}-x^{4}-12 x^{2} \ln x$, and $\mathrm{g}(x)=1+9 x-$ $9 x^{2}-x^{3}+6 x(1+x) \ln x$.
}

For ratios involving $\tau^{+} \rightarrow P^{+} \nu$ decays, the expressions are unchanged. The actual constraints [81-83] read

$$
\begin{aligned}
& R_{\mu e}^{\pi}=1+(4.1 \pm 3.3) \times 10^{-3}, \\
& R_{\tau \mu}^{\pi}=1-(5.9 \pm 5.9) \times 10^{-3}, \\
& R_{\mu e}^{K}=1-(4.8 \pm 4.7) \times 10^{-3}, \\
& R_{\tau \mu}^{K}=1-(2.2 \pm 1.4) \times 10^{-2} .
\end{aligned}
$$

All these LFU violating effects scale with $1 / m_{\mathrm{H}^{ \pm}}^{2}$ and therefore one expects that in both models, I- $g \ell \mathrm{FC}$ and II-g $\ell \mathrm{FC}$, the effects for large $m_{\mathrm{H}^{ \pm}}$are much more suppressed, including in particular the solution [A] region introduced in Sec. III. This is quite clear in the pure leptonic decays, where the most relevant constraints, Eq. (36) and $\left|g_{\mu \rightarrow e}^{S, R R}\right|$ in Eq. (37), can be comfortably satisfied, giving a contribution to the corresponding $\chi^{2}$ at a level similar to the SM. Since solution [A] corresponds to $t_{\beta} \sim 1$, the effects in semileptonic processes are similar in both models, with the effects in kaons larger by a factor of 10 than the effects in pions. The leading contribution to $R_{\mu e}^{K}-1$ is of the order of the uncertainty: since in that channel there is essentially a change of sign between the contributions in models I- $\mathrm{g} \ell \mathrm{FC}$ and II-g $\ell \mathrm{FC}$, it turns out that in the II-g $\ell \mathrm{FC}$ case the corresponding $\chi^{2}$ value can improve over the SM one, while in the $\mathrm{I}-\mathrm{g} \ell \mathrm{FC}$ case it is the other way around. In any case, for solution $[\mathrm{A}]$, these differences are small. For solution $[\mathrm{B}]$, the situation is different since we have:

$$
\text { solution [B], model I-g } \ell \mathrm{FC}, \quad \Delta_{\ell}^{K} \sim \frac{M_{K}^{2}}{m_{\ell}} \frac{n_{\ell}}{M_{H^{ \pm}}^{2} t_{\beta}},
$$

solution [B], model II-g $\ell$ FC, $\quad \Delta_{\ell}^{K} \sim-\frac{M_{K}^{2}}{m_{\ell}} \frac{n_{\ell} t_{\beta}}{M_{H^{ \pm}}^{2}}$,

considering that it requires $t_{\beta} \gg 1$ and smaller $m_{\mathrm{H}^{ \pm}}$. Clearly, lower values of $m_{\mathrm{H}^{ \pm}}$can be compensated by large values of $t_{\beta}$ in model $\mathrm{I}-\mathrm{g} \ell \mathrm{FC}$, and solution [B] is similar to [A] concerning this constraint. On the contrary, in model II-g $\ell \mathrm{FC}$, lower values of $m_{\mathrm{H}^{ \pm}}$and larger values of $t_{\beta}$ enhance the new contributions: this observable is highly relevant to eliminate solution $[\mathrm{B}]$ in model II-g $\ell$ FC.

The new scalars can also give one loop corrections to $Z \rightarrow \ell^{+} \ell^{-}$decays. In the parameter space region corresponding to solution [A], one can easily check that these new contributions are at least a factor of 30 smaller than the experimental uncertainties (in the limit $m_{\mathrm{A}}=m_{\mathrm{H}}=$ $m_{\mathrm{H}^{ \pm}} \gg M_{Z}$ they decouple, see [84]); in the parameter space of solution [B], the new contributions are larger, but still below uncertainties. 


\section{2. $b \rightarrow s \gamma$ and $B_{q}^{0}-\bar{B}_{q}^{0}$}

As loop level transitions mediated by the charged scalar, we consider contributions to the mixing in $B_{d}$ and $B_{s}$ meson systems (in particular to the dispersive part of the mixing, which controls the mass differences) and contributions to the radiative decay $b \rightarrow s \gamma$. In both cases, concerning the dependence on CKM factors of the new contributions involving $\mathrm{H}^{ \pm}$, it is clear from Table II that they are analog to their SM counterparts; this implies, for example, that there is no need to worry about new contributions to $C P$ asymmetries in $B_{d} \rightarrow J / \Psi K_{S}$ or $B_{s} \rightarrow J / \Psi \Phi$. Contributions to the mentioned mass differences in $B_{d}$ and $B_{s}$ are required to not exceed the 2-3\% level (that is already below the current level of theoretical uncertainty in the relevant matrix elements obtained from lattice QCD computations). For $b \rightarrow s \gamma$, we impose that the correction to the usual $\Gamma\left(B \rightarrow X_{s} \gamma\right)_{E_{\gamma}>1.6 \mathrm{GeV}}$ is below the experimental uncertainty. Both observables are insensitive to scalar-lepton couplings, they can only constrain $m_{\mathrm{H}^{ \pm}}$and $t_{\beta}$. For $m_{\mathrm{H}^{ \pm}}$the effect is straightforward: for large values of $m_{\mathrm{H}^{ \pm}}$, the new contributions are suppressed. Concerning $t_{\beta}$, dominant new contributions with virtual top quarks are further enhanced or suppressed by the $t_{\beta}^{-1}$ dependence in Table I: altogether, one expects that these two constraints tend to disfavor $t_{\beta} \ll 1$ and light $\mathrm{H}^{ \pm}$. We refer to [85-87] for further details.

$$
\text { F. } e^{+} e^{-} \rightarrow \mu^{+} \mu^{-}, \tau^{+} \tau^{-} \text {at LEP }
$$

LEP measured $e^{+} e^{-} \rightarrow \mu^{+} \mu^{-}, \tau^{+} \tau^{-}$with center-ofmass energies up to $\sqrt{s}=208 \mathrm{GeV}$ : although $s$-channel

contributions with virtual $\mathrm{H}$ and A do not interfere with SM $\gamma$ and $Z$ mediated contributions, for light $\mathrm{H}$, A, the resonant enhancement together with the large couplings to leptons might give predictions in conflict with data (e.g., [88]). The effect of these LEP constraints is, essentially, to forbid values of $m_{\mathrm{H}}, m_{\mathrm{A}}$ below $210-215 \mathrm{GeV}$.

\section{G. LHC searches}

We consider constraints from LHC searches of scalars, in particular,

(i) searches of dilepton resonances [89-94] which give constraints on $\sigma(p p \rightarrow S)_{[\mathrm{ggF}]} \times \mathrm{Br}\left(S \rightarrow \ell^{+} \ell^{-}\right), S=$ $\mathrm{H}, \mathrm{A}$ and $\ell=\mu, \tau$, where the production cross section $\sigma(p p \rightarrow S)_{[\mathrm{ggF}]}$ corresponds to gluon-gluon fusion,

(ii) and searches of charged scalars [95-99] which give constraints on $\sigma\left(p p \rightarrow \mathrm{H}^{ \pm} t b\right) \times \mathrm{Br}\left(\mathrm{H}^{ \pm} \rightarrow f\right)$, $f=\tau \nu, t b$.

For production, the narrow width approximation (NWA) is considered; the widths of $\mathrm{H}, \mathrm{A}$ and $\mathrm{H}^{ \pm}$can reach $\sim 10 \%$ of their respective masses: if one incorporates finite width effects through the convolution of the cross section computed in the NWA with a (relativistic) BreitWigner distribution for the scalars, the computed signal would be partially "diluted". In this sense, using the NWA is conservative since it gives stronger pointwise bounds. The constraints are incorporated as contributions of the following form in the global $\chi^{2}$ : for each "production $\times$ decay" channel with experimental bound $[\sigma \times \mathrm{Br}]_{\text {Exp }}$ and theoretical prediction $[\sigma \times \mathrm{Br}]_{\mathrm{Th}}$, the contribution is given by

$$
\chi^{2}\left([\sigma \times \mathrm{Br}]_{\mathrm{Th}}\right)= \begin{cases}0, & \text { if }[\sigma \times \mathrm{Br}]_{\mathrm{Th}} \leq 0.9 \times[\sigma \times \mathrm{Br}]_{\mathrm{Exp}} \\ 10^{3} \times\left(\frac{[\sigma \times \mathrm{Br}]_{\mathrm{Th}}}{[\sigma \times \mathrm{Br}]_{\mathrm{Exp}}}-0.9\right), & \text { if }[\sigma \times \mathrm{Br}]_{\mathrm{Th}}>0.9 \times[\sigma \times \mathrm{Br}]_{\mathrm{Exp}}\end{cases}
$$

Equation (43) is a convenient smooth approximation of a "sharp" bound/cut.

Production cross sections incorporate corrections associated to the modified fermion-scalar vertices in the following manner. For generic interaction terms

$$
\mathcal{L}_{S \bar{q} q}=-\frac{m_{t}}{v} \operatorname{St}\left(a_{t}^{S}+i b_{t}^{S} \gamma_{5}\right) t-\frac{m_{b}}{v} S \bar{b}\left(a_{b}^{S}+i b_{b}^{S} \gamma_{5}\right) b
$$

the gluon-gluon fusion production cross section reads

$$
\sigma[p p \rightarrow S]_{\mathrm{ggF}}=\sigma[p p \rightarrow S]_{[\mathrm{ggF}]}^{\mathrm{SM}-\mathrm{like}} \times \frac{\left|a_{t}^{S} m_{t} F\left(x_{t}\right)+a_{b}^{S} m_{b} F\left(x_{b}\right)\right|^{2}+\left|b_{t}^{S} m_{t} \hat{F}\left(x_{t}\right)+b_{b}^{S} m_{b} \hat{F}\left(x_{b}\right)\right|^{2}}{\left|m_{t} F\left(x_{t}\right)+m_{b} F\left(x_{b}\right)\right|^{2}},
$$

with $x_{q} \equiv\left(m_{q} / m_{S}\right)^{2}$, and $F(x)$ and $\hat{F}(x)$ the loop functions corresponding to scalar or pseudoscalar couplings, respectively; $\sigma[p p \rightarrow S]_{[\mathrm{ggF}]}^{\mathrm{SM}-\mathrm{like}}$ can be found in [100-103]. This simple recipe also gives sufficiently good agreement with results for a SM-Higgs-like neutral pseudoscalar, which can be found in [103-106]. The couplings in Eq. (44) for $S=\mathrm{H}$, A in each model can be read in Table I.

Similarly, for the production cross sections $p p \rightarrow H^{ \pm} t b$ (i.e., $\mathrm{H}^{ \pm}$in association with $t b$ ), we refer to $[107,108]$, which provide results, labeled here $\sigma_{[\operatorname{Ref}]}$, for a type II $2 \mathrm{HDM}$ with $t_{\beta}=1$. For arbitrary values of $t_{\beta}$, we use 


$$
\begin{aligned}
& \text { Model I-g } \ell \mathrm{FC}: \sigma_{\mathrm{I}}\left(t_{\beta}\right)=\frac{\left(m_{t} / t_{\beta}\right)^{2}+\left(m_{b} / t_{\beta}\right)^{2}}{m_{t}^{2}+m_{b}^{2}} \times \sigma_{[\operatorname{Ref}]}=\frac{1}{t_{\beta}^{2}} \sigma_{[\operatorname{Ref}]}, \\
& \text { Model II-g } \ell \mathrm{FC}: \sigma_{\mathrm{II}}\left(t_{\beta}\right)=\frac{\left(m_{t} / t_{\beta}\right)^{2}+\left(m_{b} t_{\beta}\right)^{2}}{m_{t}^{2}+m_{b}^{2}} \times \sigma_{[\operatorname{Ref}]}=\frac{1}{t_{\beta}^{2}} \frac{1+t_{\beta}^{4} m_{b}^{2} / m_{t}^{2}}{1+m_{b}^{2} / m_{t}^{2}} \sigma_{[\operatorname{Ref}]} .
\end{aligned}
$$

As an additional check, (i) the previous cross sections and (ii) the computations of the decay branching ratios of the scalars, have been compared with the results of MadGraph5 AMC@NLO [109] at leading order. With FeynRules [110] and NLOCT [111,112], the needed universal Feynrules Output at next to leading order (NLO) of the $\mathrm{I}-\mathrm{g} \ell \mathrm{FC}$ and $\mathrm{II}-\mathrm{g} \ell \mathrm{FC}$ models is produced. A good agreement in the gluon-gluon fusion production cross section is found, given the fact that the MadGraph5_AMC@NLO calculation is at leading order (one loop in this case). For the branching ratios, there is complete agreement.

\section{RESULTS}

As discussed in Sec. III, we expect, at least, two different types of solution to the $\delta a_{\ell}$ anomalies. In the following we refer to them, as anticipated, as solutions [A] and [B]. Solution [A] corresponds to $t_{\beta} \sim 1$, heavy neutral new scalars (with masses in the $1-2 \mathrm{TeV}$ range), and both anomalies explained by two loop Barr-Zee contributions. Solution [B] corresponds instead to large $t_{\beta}$, lighter new scalars, with $\delta a_{e}$ obtained through two loop Barr-Zee contributions while in $\delta a_{\mu}$ the most important contributions are one loop and H-mediated. Note that in general one would expect a set of intermediate solutions between [A] and $[\mathrm{B}]$, at least in model $\mathrm{I}-\mathrm{g} \ell \mathrm{FC}$, where we have $a$ priori identified the presence of both solutions. For model II-g $\ell \mathrm{FC}$ we can only anticipate with some certainty the presence of solution [A].

It is therefore very important to find out which constraints, if any, can distinguish among both types of solutions. One should also remember that quite large couplings of the new scalars to leptons are required to explain the anomalies. This fact confers a special role to dilepton resonance and charged scalar searches at the LHC. Consequently the analyses are separated in two stages: (i) one, labeled "No LHC", which includes all constraints discussed in Sec. IV except for the LHC searches which are not imposed as constraints, and (ii) the complete analysis with all constraints, including these LHC searches.

One should also remark, before presenting results, that solutions [A] and [B] as discussed above, cannot be realized when the scalar potential in Eq. (13) is exactly $\mathbb{Z}_{2}$ symmetric, i.e., when $\mu_{12}^{2}=0$. This was to be expected. The reason to have difficulties obtaining solution [A] with the exactly $\mathbb{Z}_{2}$ symmetric potential is simple: it does not allow a "decoupling regime" $[67,113,114]$, i.e., in that case one cannot have scalars heavier than $\sim 1 \mathrm{TeV}$ (without violating requirements such as perturbativity). On the other hand, concerning solution [B], the exact $\mathbb{Z}_{2}$ symmetry does not allow large $t_{\beta}$. Introducing $\mu_{12}^{2} \neq 0$ removes both obstacles. In the plots to follow, the results from the "No LHC" analysis correspond to lighter red regions while the results from the full analysis correspond to darker blue regions. The regions represented are allowed at $2 \sigma$ (for a $2 D-\chi^{2}$ distribution); the $\chi^{2}$ or likelihood function used in the numerical analysis implements the constraints of Sec. IV.

In Fig. 3 we have $\operatorname{Re}\left(n_{\mu}\right)$ versus $\operatorname{Re}\left(n_{e}\right)$; the full analysis shows, clearly, three disjointed regions. As indicated in the figure, the bottom left small region corresponds to solution [A], and reproduces the linear relation of Eq. (28), arising from the explanation of both anomalies through two loop Barr-Zee contributions. The largest blue region to the bottom right corresponds to solution [B] with $\operatorname{Re}\left(n_{\mu}\right)<0$, where $\delta a_{e}$ is two loop dominated while $\delta a_{\mu}$ also receives significant one loop contributions. In this region there is no linear relation among $\operatorname{Re}\left(n_{e}\right)$ and $\operatorname{Re}\left(n_{\mu}\right)$. For $\operatorname{Re}\left(n_{\mu}\right)>0$, solution [B] corresponds to the top blue region [the subindex \pm in $\left[\mathrm{B}_{ \pm}\right]$refers to the sign of $\left.\operatorname{Re}\left(n_{\mu}\right)\right]$. It is clear, from the underlying red region, that excluding LHC searches, there is a smooth transition between solutions [A] and $\left[\mathrm{B}_{-}\right]$where all kinds of contributions must be considered: we recall that the numerical analyses incorporate the

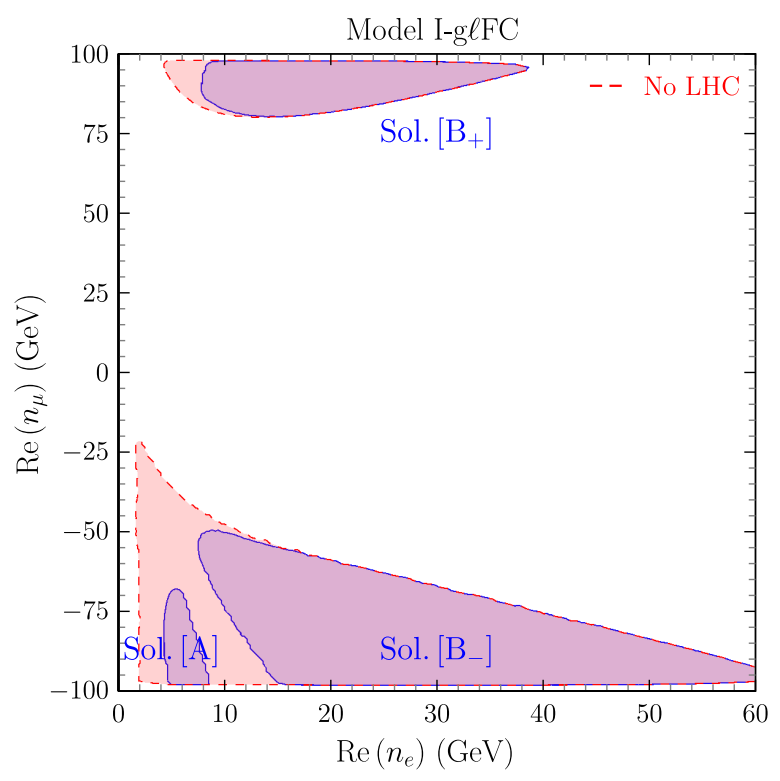

FIG. 3. $\operatorname{Re}\left(n_{\mu}\right)$ vs $\operatorname{Re}\left(n_{e}\right)$. 


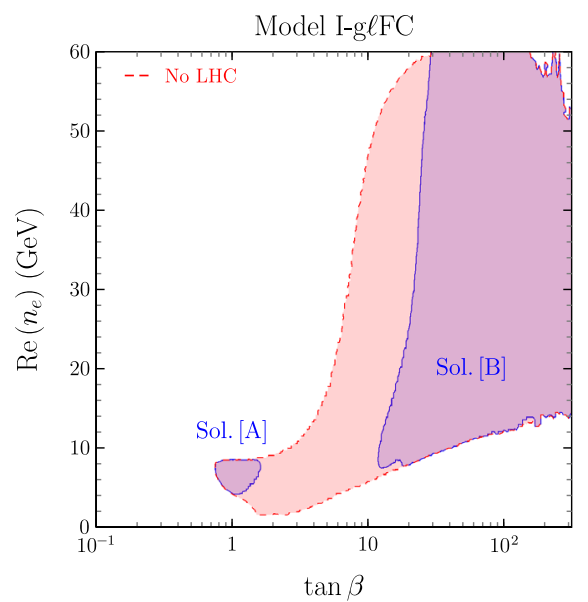

(a)

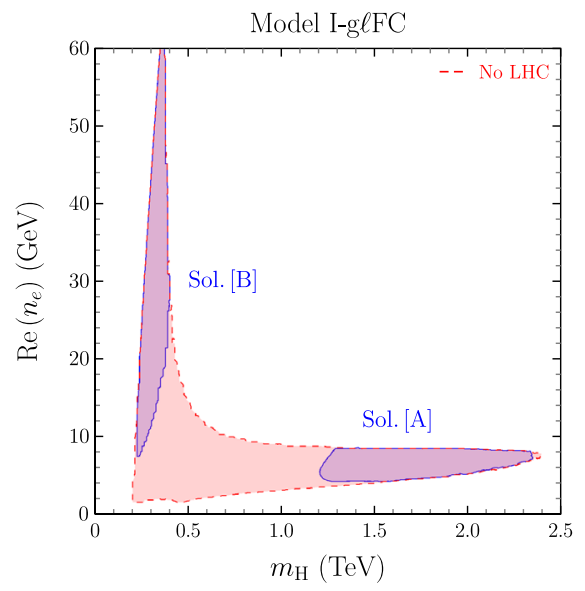

(d)

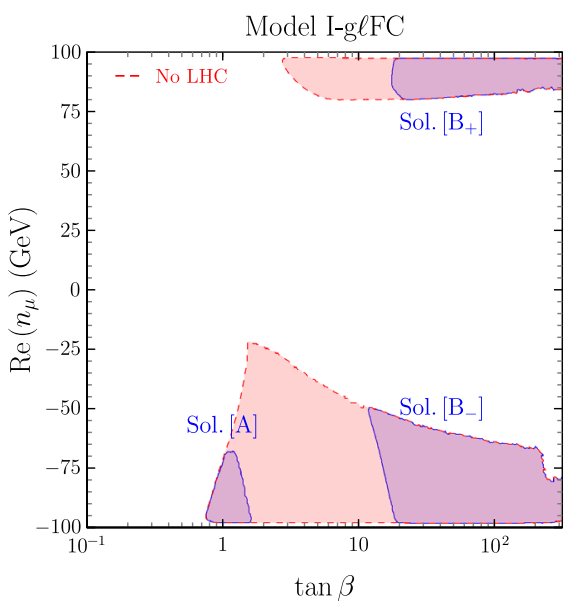

(b)

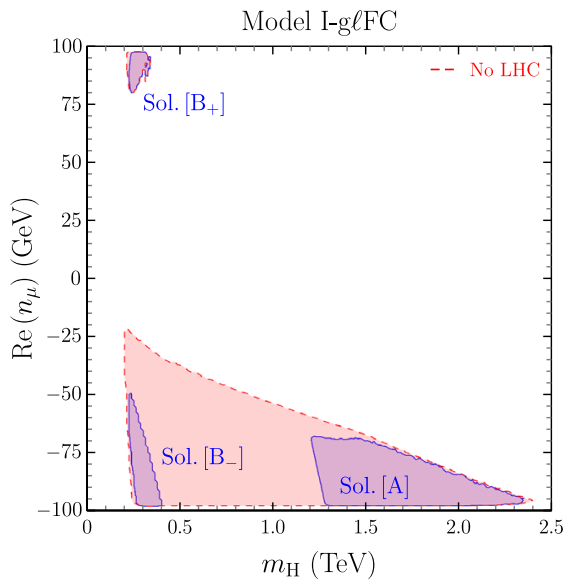

(e)

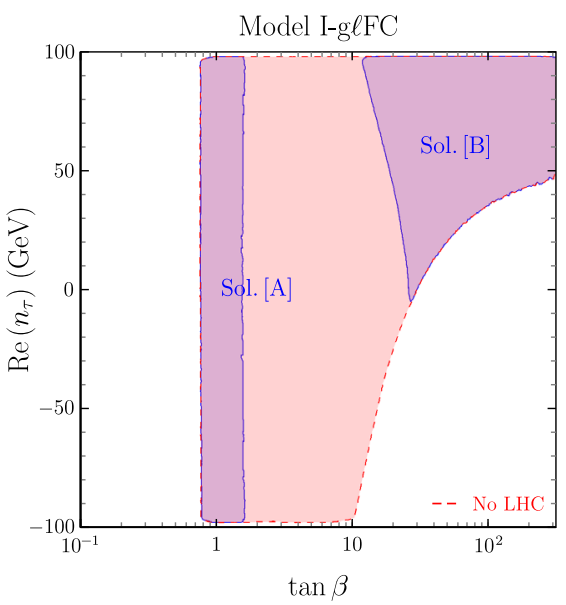

(c)

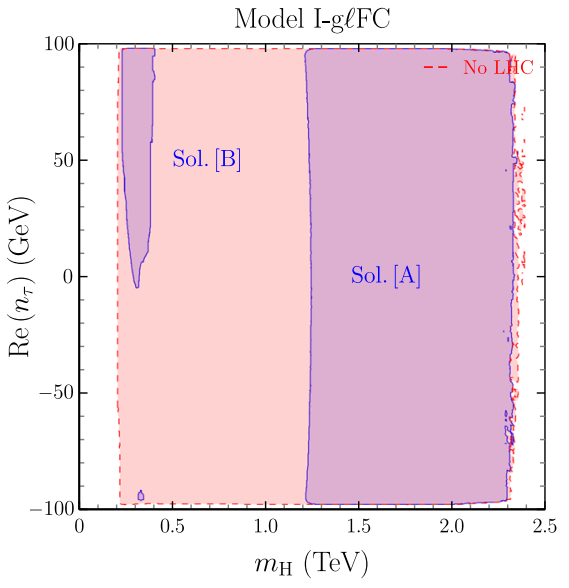

(f)

FIG. 4. $n_{\ell}$ couplings versus $t_{\beta}, m_{\mathrm{H}}$.

complete expressions of Appendix B, which consider one and two loop contributions with all possible fermions in the fermion loop of Barr-Zee terms. It is important to stress that, since the lepton couplings to $\mathrm{H}$ and $\mathrm{A}$ can be quite large, it is mandatory to include all leptons in the computation of Barr-Zee terms.

Figure 4 shows results for $\operatorname{Re}\left(n_{\ell}\right)$ versus $t_{\beta}$ and $m_{\mathrm{H}}$. From previous discussions, the regions corresponding to solutions $[\mathrm{A}]$ and $[\mathrm{B}]$ can be easily identified. For example, in Fig. 4(a), the blue region reaching larger values of $\operatorname{Re}\left(n_{e}\right)$, with $t_{\beta} \geq 13$ and $200 \mathrm{GeV} \leq m_{\mathrm{H}} \leq 370 \mathrm{GeV}$ is clearly associated to solution [B]. Figures 4(b) and 4(e) illustrate the same aspects regarding now $\operatorname{Re}\left(n_{\mu}\right)$. For $\operatorname{Re}\left(n_{\tau}\right)$ it also follows from Figures 4(c) and 4(f) that $\operatorname{Re}\left(n_{\tau}\right)>0$ is required in solution [B] (one can indeed check that it gives a subdominant but necessary two loop contribution to obtain the appropriate value of $\delta a_{\mu}$ ).

To characterize more precisely solutions [A] and [B], Fig. 5 shows correlations among scalar masses and with $t_{\beta}$. In particular, it is clear that in solution [A] all new scalars are heavy, with masses in the 1.2-2.3 TeV range, and mass differences not exceeding $\pm 200 \mathrm{GeV}$. For solution [B], some important results can be observed: (i) in addition to the existence of separate regions $\left[\mathrm{B}_{+}\right]$and $\left[\mathrm{B}_{-}\right]$for both signs of $\operatorname{Re}\left(n_{\mu}\right)$, there are two separate manners in which solution [B] can arise, one region where $m_{\mathrm{H}^{ \pm}} \in$ $[0.4 ; 0.9] \mathrm{TeV}$ and $m_{\mathrm{A}}=m_{\mathrm{H}^{ \pm}}$to a high degree of accuracy and another smaller region where $m_{\mathrm{H}^{ \pm}} \in[0.25 ; 0.35] \mathrm{TeV}$ and $m_{\mathrm{H}}=m_{\mathrm{H}^{ \pm}}$to a high degree of accuracy; (ii) in all cases, $m_{\mathrm{H}}<m_{\mathrm{A}}$. This last inequality, as analyzed later, must allow the decay $\mathrm{A} \rightarrow \mathrm{HZ}$ (additionally, either $\mathrm{H}^{ \pm} \rightarrow$ $\mathrm{H} W^{ \pm}$or $\mathrm{A} \rightarrow \mathrm{H}^{ \pm} W^{\mp}$ would also be allowed); together with the electroweak precision constraints (in particular the oblique parameter $T$ ), this forces either $m_{\mathrm{A}}=m_{\mathrm{H}^{ \pm}}$or $m_{\mathrm{H}}=m_{\mathrm{H}^{ \pm}}$. These two results match nicely with the need for $\mathrm{H}$ to be as light as possible (LEP constraints will force in any case $m_{\mathrm{H}} \geq 210 \mathrm{GeV}$ ) in order to produce the main contribution (at one loop) to $\delta a_{\mu}$.

Figure 6 shows the resonant $[p p]_{\mathrm{ggF}} \rightarrow \mathrm{S} \rightarrow \mu^{+} \mu^{-}$cross sections with respect to $m_{\mathrm{S}}$ for $\mathrm{S}=\mathrm{H}, \mathrm{A}$. The black line 


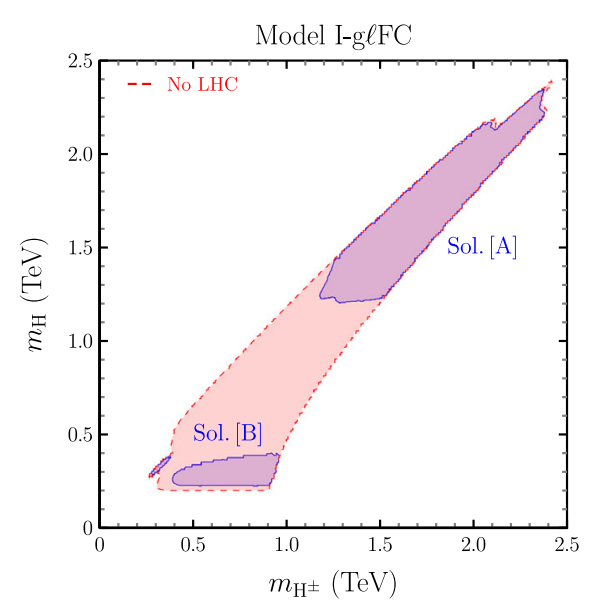

(a)

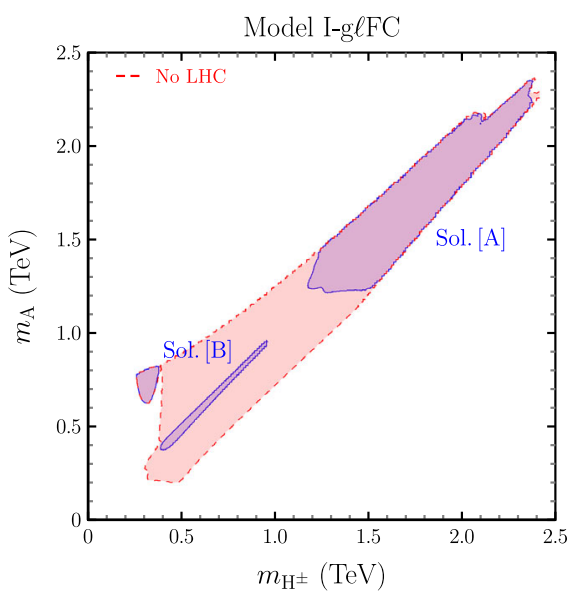

(b)

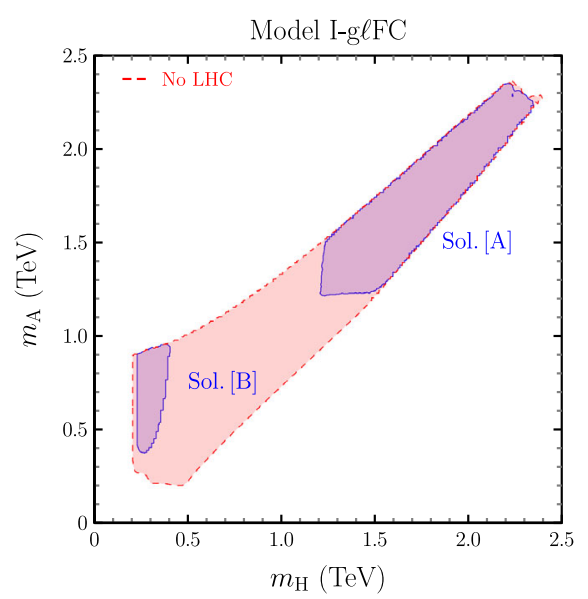

(c)

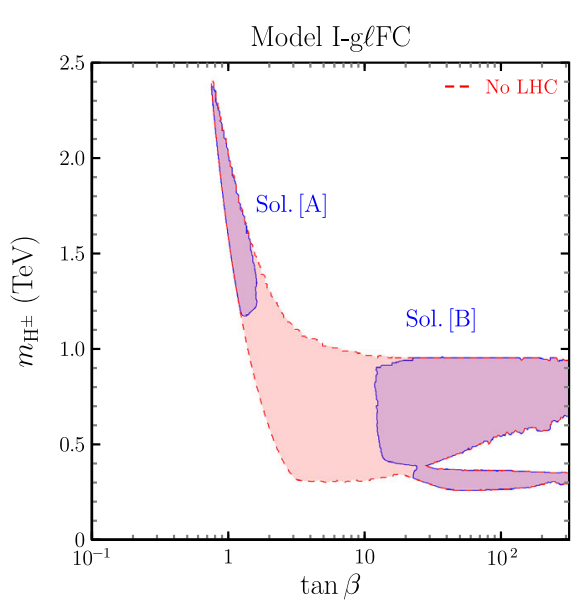

(d)

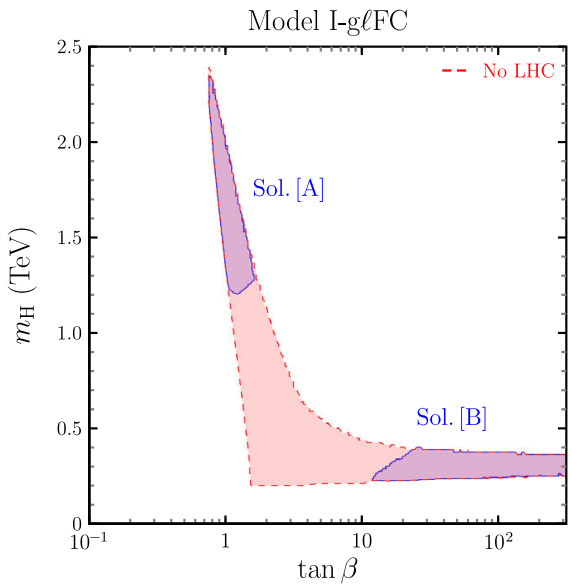

(e)

FIG. 5. Scalar sector.

shows the LHC bounds included in the full analysis. In gluon-gluon fusion production, for the same scalar mass, the gluon-gluon-pseudoscalar amplitude is 2-6 times larger than the corresponding gluon-gluon-scalar amplitude (that is $2^{2}-6^{2}$ larger pseudoscalar vs scalar production cross sections). One could have expected, attending to this fact, that the constraints from LHC searches on $\sigma(p p \rightarrow$ $\mathrm{A})_{\mathrm{ggF}} \times \operatorname{Br}\left(\mathrm{A} \rightarrow \mu^{+} \mu^{-}\right)$versus $m_{\mathrm{A}}$ would be responsible for the separation among solutions $[\mathrm{A}]$ and $[\mathrm{B}]$. Figure 6(a) disproves this naive expectation, as Fig. 6(b) shows it is rather $\sigma(p p \rightarrow \mathrm{H})_{\mathrm{ggF}} \times \mathrm{Br}\left(\mathrm{H} \rightarrow \mu^{+} \mu^{-}\right)$which shows how the bounds from LHC searches separate the solutions by excluding $m_{\mathrm{H}} \in[380 ; 1200] \mathrm{GeV}$ (i.e., eliminating the red region "bridge" connecting the blue regions). Comparing the shape of the allowed regions in Figures 6(a) and 6(b) it is also clear that, besides the production cross section, the branching ratios $\mathrm{Br}\left(\mathrm{H}, \mathrm{A} \rightarrow \mu^{+} \mu^{-}\right)$may play an important role.

On this respect, let us start by observing that, since values of $\left|\operatorname{Re}\left(n_{\mu}\right)\right|$ larger than some minimal $\left|\operatorname{Re}\left(n_{\mu}\right)\right|_{\text {Min }}$ are required to explain $\delta a_{\mu}$, both $\operatorname{Br}\left(\mathrm{H} \rightarrow \mu^{+} \mu^{-}\right)$and $\operatorname{Br}\left(\mathrm{A} \rightarrow \mu^{+} \mu^{-}\right)$are bounded from below. The dominant decay channels of the new scalars are shown in Fig. 8; Figures 8(b) and 8(f) show that $\operatorname{Br}\left(\mathrm{H} \rightarrow \mu^{+} \mu^{-}\right)$and $\operatorname{Br}\left(\mathrm{A} \rightarrow \mu^{+} \mu^{-}\right)$are indeed bounded from below, but in the case of $\mathrm{H} \rightarrow \mu^{+} \mu^{-}$the lower bound is larger than that of $\mathrm{A} \rightarrow \mu^{+} \mu^{-}$(it can even saturate the decay width of $\mathrm{H}$ ). This explains the narrowness of the red and blue regions in Fig. 6(b) for $m_{\mathrm{H}}>400 \mathrm{GeV}$. One should keep in mind that solutions $[\mathrm{A}]$ and $[\mathrm{B}]$ also differ quite substantially in the values of $t_{\beta}$ : in Fig. 5(e) it is clear that large $m_{\mathrm{H}}>$ $1 \mathrm{TeV}$ requires $t_{\beta} \sim 1$, while $m_{\mathrm{H}}<500 \mathrm{GeV}$ is compatible with a broad range $t_{\beta} \in\left[1 ; 10^{2}\right]$. This is the last ingredient necessary to interpret the shape of Fig. 6(b). For $m_{\mathrm{H}}<500 \mathrm{GeV}$, without constraints from LHC searches, the broad range of $t_{\beta}$ values gives a broad range for $\sigma(p p \rightarrow \mathrm{H})_{\mathrm{ggF}}$ : since the gluon-gluon fusion production cross section is proportional to $t_{\beta}^{-2}$, and thus for solution [B] there is a substantial suppression of $\sigma(p p \rightarrow \mathrm{H})_{\mathrm{ggF}}$ due to $t_{\beta} \gg 1$. Due to the larger production cross section of a 


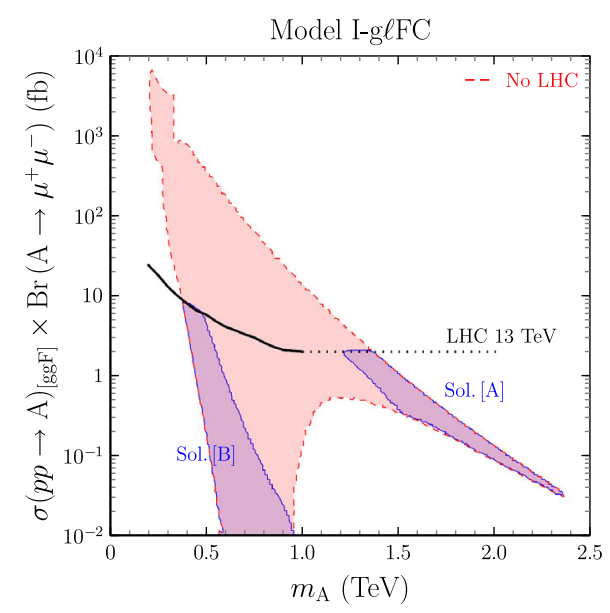

(a)

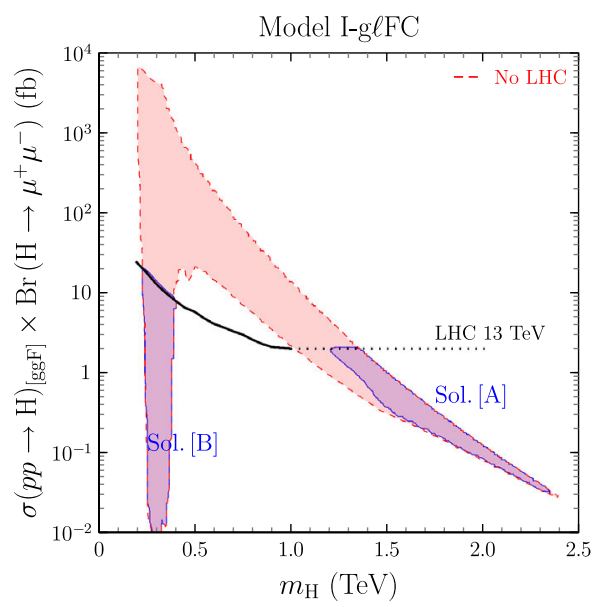

(b)

FIG. 6. $\quad[p p]_{\mathrm{ggF}} \rightarrow \mathrm{S} \rightarrow \mu^{+} \mu^{-}$versus $m_{\mathrm{S}}$.

pseudoscalar, despite the $t_{\beta}^{-2}$ suppression, LHC searches might rule out $p p \rightarrow \mathrm{A} \rightarrow \mu^{+} \mu^{-}$predictions for solution [B]: as Fig. 6(a) shows, that is not the case. This is clearly achieved through a reduction of $\operatorname{Br}\left(\mathrm{A} \rightarrow \mu^{+} \mu^{-}\right)$; Figures 8(f) and 8(e) show that $\mathrm{A} \rightarrow \mathrm{HZ}$ contributes decisively to reduce $\operatorname{Br}\left(\mathrm{A} \rightarrow \mu^{+} \mu^{-}\right)$, evade LHC bounds, and obtain a viable solution [B]. For this reason, as anticipated, $m_{\mathrm{A}}>m_{\mathrm{H}}+M_{\mathrm{Z}}$. For the charged scalar $\mathrm{H}^{ \pm}$, the behavior of the most relevant decay channels $\mathrm{H}^{+} \rightarrow \mu^{+} \nu, \tau^{+} \nu, t \bar{b}, \mathrm{H} W^{ \pm}$mirrors the corresponding $\mathrm{A} \rightarrow \mu^{+} \mu^{-}, \tau^{+} \tau^{-}, t \bar{t}, \mathrm{HZ}$, as Figures 8(j)-8(i) show. The only minor difference arises for solution $[\mathrm{B}]$ in the small region where $m_{\mathrm{H}^{ \pm}} \simeq m_{\mathrm{H}}$ : in that region, (i) $\mathrm{H}^{ \pm} \rightarrow \mathrm{H} W^{ \pm}$is forbidden and (ii) in addition to $\mathrm{A} \rightarrow \mathrm{HZ}$, also $\mathrm{A} \rightarrow \mathrm{H}^{ \pm} W^{\mp}$ (not shown) has a large branching ratio.

Figure 7 shows that resonant $\tau^{+} \tau^{-}$searches are less constraining than the corresponding $\mu^{+} \mu^{-}$searches in
Fig. 6. Concerning production of $\mathrm{H}^{ \pm}$, Fig. 9 shows that current results from searches at the LHC are much less constraining than the results from resonant dilepton searches in Figs. 7 and 6. Results in the previous figures concern model I- $\mathrm{g} \ell \mathrm{FC}$, where, in addition to solution [A] in which both $\delta a_{e}$ and $\delta a_{\mu}$ arise from 2 loop contributions, a second set of solutions [B] exists in which 1 loop contributions are dominant in $\delta a_{\mu}$. For model II-g $\ell$ FC this second possibility is not available, and only solution $[\mathrm{A}]$ is obtained. Furthermore, since $t_{\beta} \sim 1$ in solution [A], the corresponding allowed regions do not differ much in both models I-g $\ell \mathrm{FC}$ and II-g $\ell \mathrm{FC}$. We do not show figures corresponding to model II-g $\ell$ FC since the allowed regions in that case very approximately coincide with "Sol. [A]" regions in model $\mathrm{I}-\mathrm{g} \ell \mathrm{FC}$ plots.

Finally, Fig. 10 illustrates with some examples the kind of clear signal that solution $[\mathrm{B}]$ in model $\mathrm{I}-\mathrm{g} \ell \mathrm{FC}$ gives in

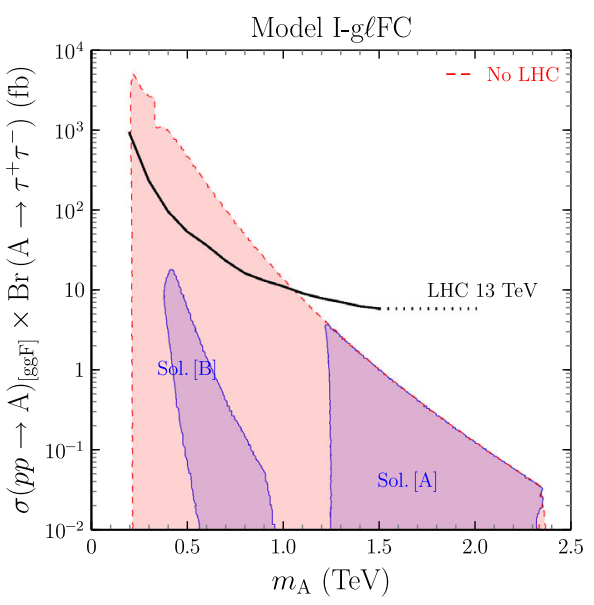

(a)

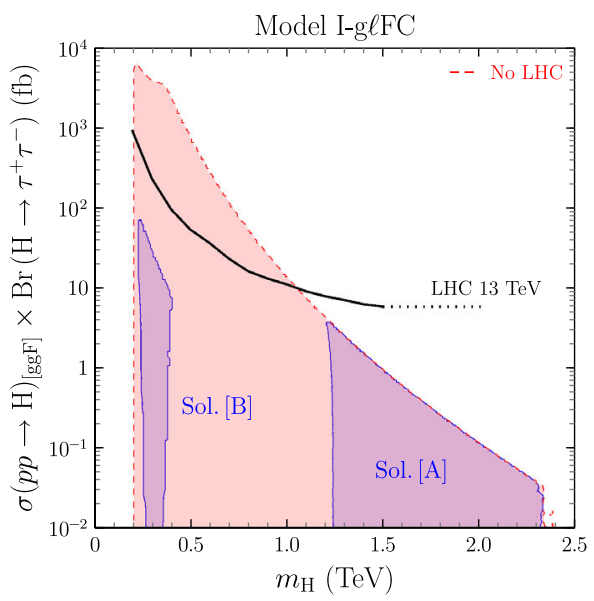

(b)

FIG. 7. $[p p]_{\mathrm{ggF}} \rightarrow \mathrm{S} \rightarrow \tau^{+} \tau^{-}$versus $m_{\mathrm{S}}$. 


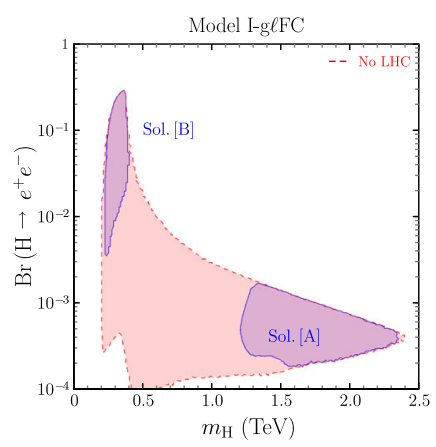

(a)

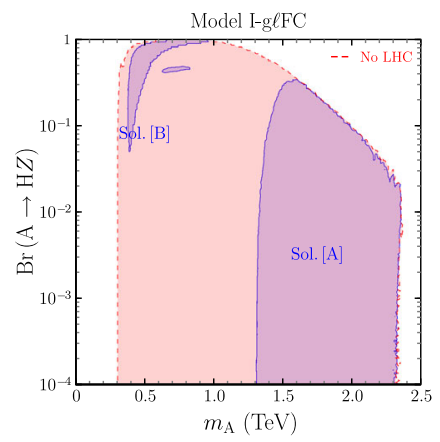

(e)

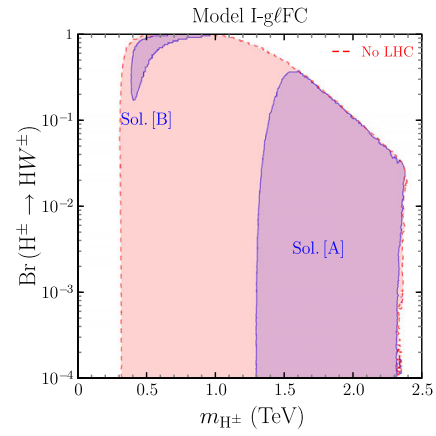

(i)

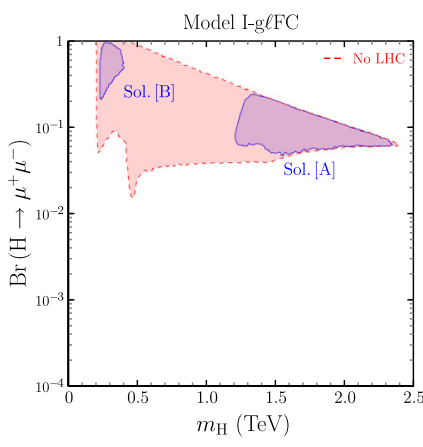

(b)

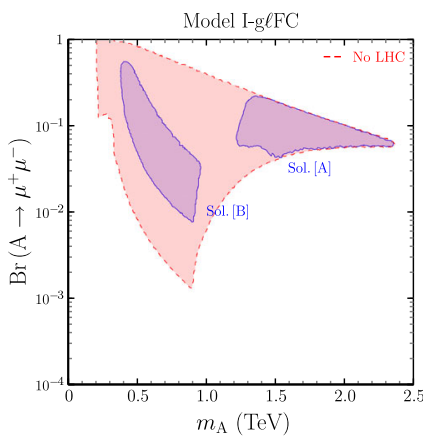

(f)

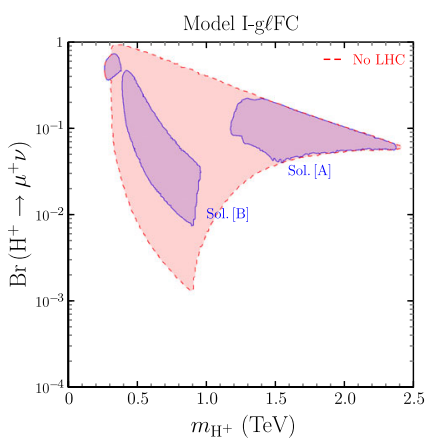

(j)

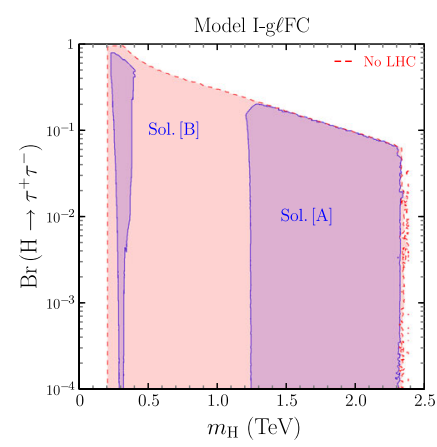

(c)

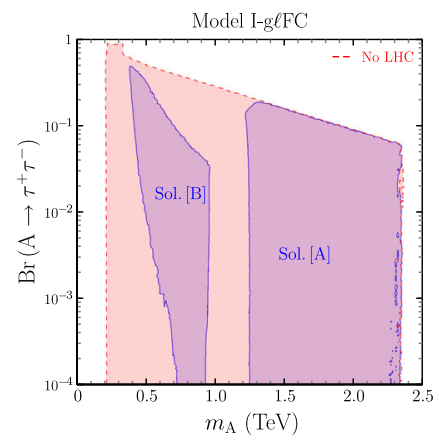

(g)

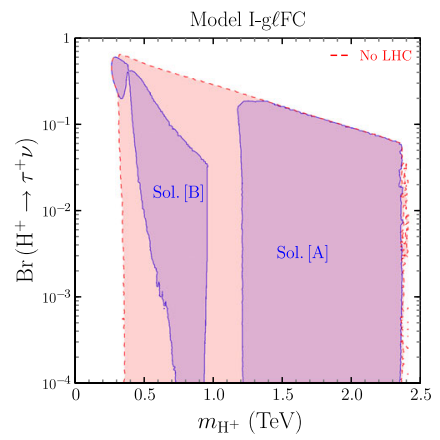

(k)

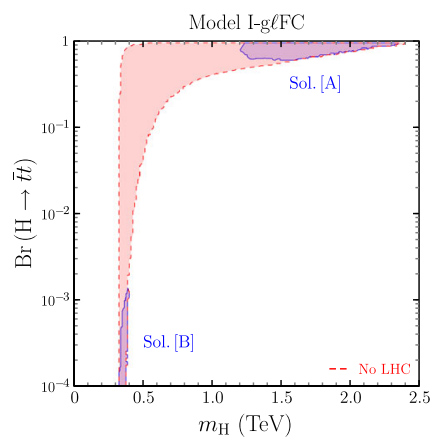

(d)

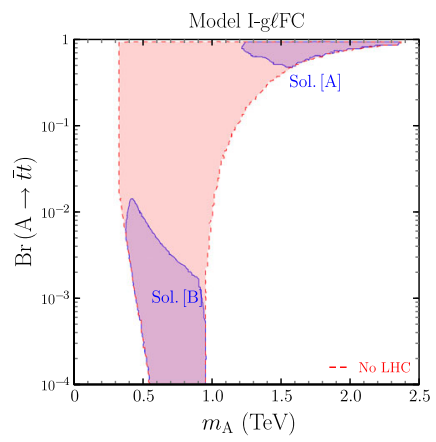

(h)

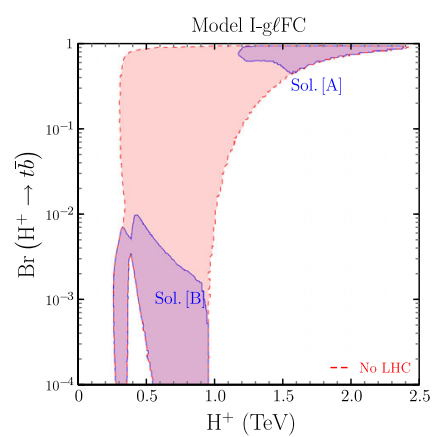

(l)

FIG. 8. Dominant decay channels of $\mathrm{H}, \mathrm{A}, \mathrm{H}^{ \pm}$.

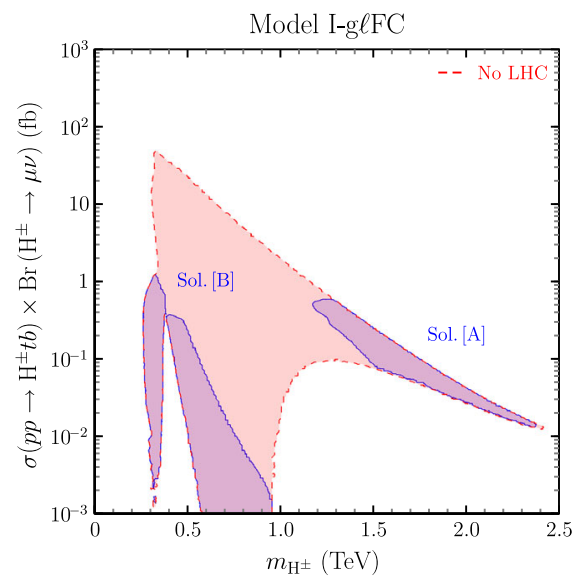

(a)

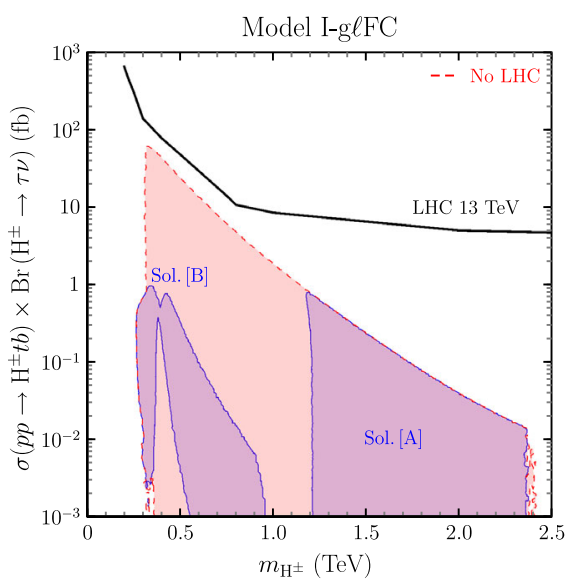

(b)

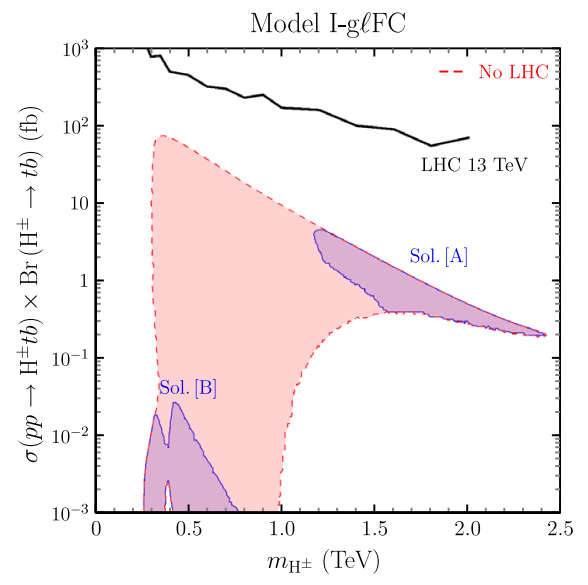

(c)

FIG. 9. $\quad p p \rightarrow \mathrm{H}^{ \pm}(t b) \rightarrow \ell \nu, t b$ versus $m_{\mathrm{H}^{ \pm}}$. 


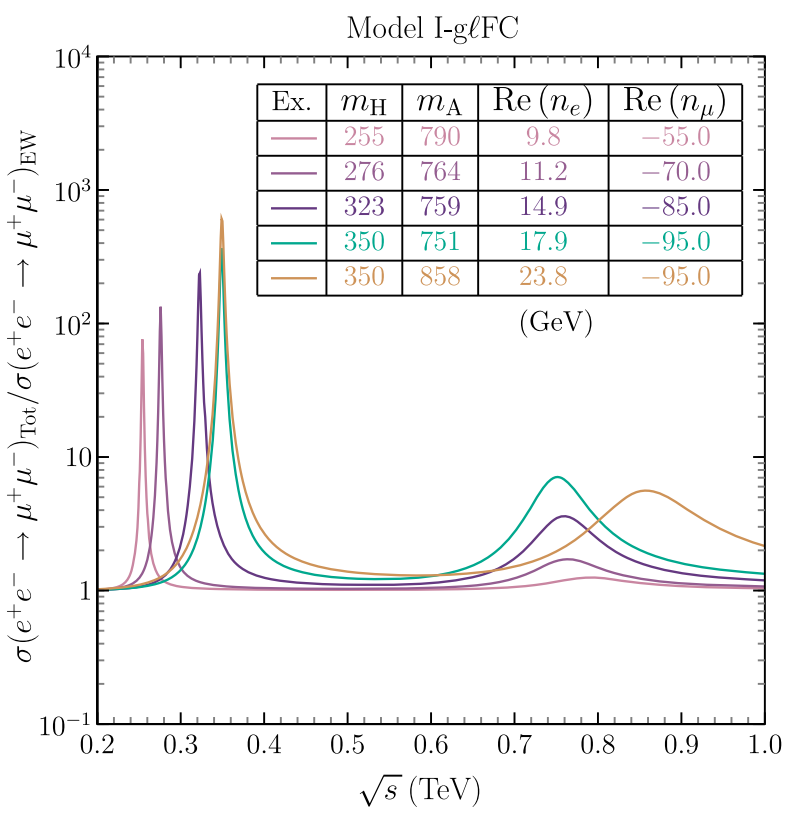

FIG. 10. $e^{+} e^{-} \rightarrow \mu^{+} \mu^{-}$for $\sqrt{s} \in[0.2 ; 1.0] \mathrm{TeV}$, examples of solution [B] in model I-g $\ell$ FC.

$e^{+} e^{-} \rightarrow \mu^{+} \mu^{-}$scattering at energies beyond the range explored at LEP.

\section{CONCLUSIONS}

General 2HDMs without SFCNC in the lepton sector are a robust framework, stable under renormalization group evolution, in which the possibility of decoupling the electron, muon, and tau interactions is open. In this context, lepton flavor universality is broken beyond the mass proportionality, and a different behavior among charged leptons can be accommodated in a simple way, without introducing highly constrained SFCNC. We have considered two of these general flavor conserving models in the leptonic sector, to address simultaneously the electron and muon $(g-2)$ anomalies. These two models, I-g $\ell \mathrm{FC}$ and II-g $\ell \mathrm{FC}$, differ in the quark Yukawa couplings, which coincide, respectively, with the ones in type I and in type II 2HDMs. There are two types of solutions that fully reproduce both the muon and electron $(g-2)$ anomalies, while remaining in agreement with constraints from LEP and LHC, from LFU, from flavor and electroweak physics, and theoretical requirements in the scalar sector. In one solution, all the new scalars have masses in the $1-2.5 \mathrm{TeV}$ range, the vevs of both doublets are quite similar and both anomalies are dominated by two loop Barr-Zee contributions. This solution arises in both models, $\mathrm{I}-$ and II- $\mathrm{g} \ell \mathrm{FC}$. There is a second type of solution, where one loop contributions are dominant in the muon anomaly, the new scalars have masses below $1 \mathrm{TeV}$, and the vevs quite different, with a ratio in the range 10-100. Among the new scalars, the second neutral one $\mathrm{H}$ is the lighter, with a mass in the range $210-390 \mathrm{GeV}$, while the pseudoscalar $\mathrm{A}$ is the heavier, with a mass in the range $400-900 \mathrm{GeV}$. The new charged scalar $\mathrm{H}^{ \pm}$is almost degenerate either with the scalar or with the pseudoscalar. This solution is only available in the I-g $\ell \mathrm{FC}$ model. In both solutions, soft breaking of the $\mathbb{Z}_{2}$ symmetry of the Higgs potential is required, together with lepton Yukawa couplings with values from 1 to $100 \mathrm{GeV}$. These results imply for LHC searches, in the light scalar solution, that it should be easier to find both charged and neutral Higgses in the muonic channel. The heavy channels, like the top quark channels, are more suited to searches addressing the heavy scalars solution.

\section{ACKNOWLEDGMENTS}

The authors thank Luca Fiorini and Marcel Vos for useful discussions. The authors acknowledge support from Spanish Grant No. FPA2017-85140-C3-3-P (AEI/ FEDER, UE) and No. PROMETEO 2019-113 (Generalitat Valenciana). The work of F. C. G. is funded by Ministerio de Ciencia, Innovacion y Universidades, Spain (Grant No. BES-2017-080070) and partially supported by a ShortTerm Scientific Mission Grant from the COST Action CA15108. M. N. acknowledges support from Fundação para a Ciência e a Tecnologia (FCT, Portugal) through the following projects: CFTP-FCT Unit 777 (UID/FIS/ 00777/2013, UID/FIS/00777/2019) and PTDC/FIS-PAR/ 29436/2017, partially funded through POCTI (FEDER), COMPETE, QREN, and EU.

\section{APPENDIX A: YUKAWA COUPLINGS}

For completeness we show in this Appendix the form of the Yukawa couplings in the general case with arbitrary scalar mixing $\mathcal{R}$ and couplings $\mathrm{N}_{d}, \mathrm{~N}_{u}, \mathrm{~N}_{\ell}$. For neutral scalars they read $^{5}$

$$
\begin{aligned}
\mathcal{L}_{\mathrm{S} \bar{f} f}= & -\frac{\mathrm{S}}{\bar{v}} \bar{f}\left[\mathcal{R}_{1 s} \mathrm{M}_{f}+\mathcal{R}_{2 s} \frac{\mathrm{N}_{f}+\mathrm{N}_{f}^{\dagger}}{2}+i \epsilon_{(f)} \mathcal{R}_{3 s} \frac{\mathrm{N}_{f}-\mathrm{N}_{f}^{\dagger}}{2}\right] f \\
& -\frac{\mathrm{S}}{v} \bar{f}_{5}\left(\mathcal{R}_{2 s} \frac{\mathrm{N}_{f}-\mathrm{N}_{f}^{\dagger}}{2}+i \epsilon_{(f)} \mathcal{R}_{3 s} \frac{\mathrm{N}_{f}+\mathrm{N}_{f}^{\dagger}}{2}\right) f
\end{aligned}
$$

where $s=1,2,3$ in correspondence with $\mathrm{S}=\mathrm{h}, \mathrm{H}, \mathrm{A}$; $f=u, d, \ell$, and, in terms proportional to $\mathcal{R}_{3 s}, \epsilon_{(d)}=$ $\epsilon_{(\ell)}=-\epsilon_{(u)}=1$.

The Yukawa couplings of $\mathrm{H}^{ \pm}$read

$$
\begin{aligned}
\mathcal{L}_{\mathrm{H}^{ \pm} u d}= & \frac{\mathrm{H}^{-}}{\sqrt{2} v} \bar{d}\left[V^{\dagger} \mathrm{N}_{u}-\mathrm{N}_{d}^{\dagger} V^{\dagger}+\gamma_{5}\left(V^{\dagger} \mathrm{N}_{u}+\mathrm{N}_{d}^{\dagger} V^{\dagger}\right)\right] u \\
& +\frac{\mathrm{H}^{+}}{\sqrt{2} v} \bar{u}\left[\mathrm{~N}_{u}^{\dagger} V-V \mathrm{~N}_{d}+\gamma_{5}\left(\mathrm{~N}_{u}^{\dagger} V+V \mathrm{~N}_{d}\right)\right] d,
\end{aligned}
$$

\footnotetext{
${ }^{5}$ Flavor indices are omitted for simplicity: e.g., $\mathrm{M}_{f}$ is the diagonal mass matrix.
} 

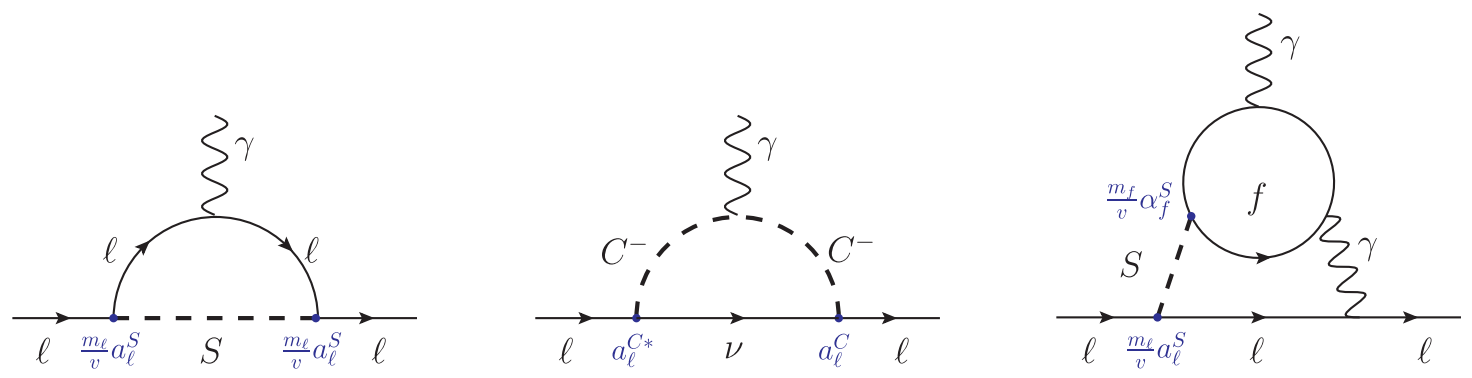

FIG. 11. Illustrative 1 and 2 loop contributions to $\delta a_{\ell}$.

and

$\mathcal{L}_{\mathrm{H}^{ \pm} \ell \nu}=-\frac{\sqrt{2}}{v} \mathrm{H}^{+} \bar{\nu}_{L} U^{\dagger} \mathrm{N}_{\ell} \ell_{R}-\frac{\sqrt{2}}{v} \mathrm{H}^{-} \bar{\ell}_{R} \mathrm{~N}_{\ell}^{\dagger} U \nu_{L}$.

$V$ and $U$ are, respectively, the CKM and PMNS mixing matrices. ${ }^{6}$

\section{APPENDIX B: CONTRIBUTIONS TO $(g-2)_{\ell}$}

\section{One loop contributions}

Yukawa interactions (of neutral scalars $S$ ) of the form

$$
\mathcal{L}_{S \ell \ell}=-\frac{m_{\ell}}{v} S \bar{\ell}\left(a_{\ell}^{S}+i b_{\ell}^{S} \gamma_{5}\right) \ell
$$

give one loop contributions to the anomalous magnetic moment of lepton $\ell$ of the form

$$
\begin{aligned}
\Delta a_{\ell}^{(1)}= & \frac{1}{8 \pi^{2}} \frac{m_{\ell}^{2}}{v^{2}} \sum_{S}\left\{\left[a_{\ell}^{S}\right]^{2}\left(2 I_{2}\left(x_{\ell S}\right)-I_{3}(x)\right)\right. \\
& \left.-\left[b_{\ell}^{S}\right]^{2} I_{3}\left(x_{\ell S}\right)\right\},
\end{aligned}
$$

with $x_{\ell S} \equiv m_{\ell}^{2} / m_{S}^{2}$ and

$I_{2}(x)=1+\frac{1-2 x}{2 x \sqrt{1-4 x}} \ln \left(\frac{1+\sqrt{1-4 x}}{1-\sqrt{1-4 x}}\right)+\frac{1}{2 x} \ln x$,

$$
\begin{aligned}
I_{3}(x)= & \frac{1}{2}+\frac{1}{x}+\frac{1-3 x}{2 x^{2} \sqrt{1-4 x}} \ln \left(\frac{1+\sqrt{1-4 x}}{1-\sqrt{1-4 x}}\right) \\
& +\frac{1-x}{2 x^{2}} \ln x .
\end{aligned}
$$

For $x \ll 1$,

${ }^{6}$ Equation (A3) assumes massless neutrinos, in which case one can indeed set $U \rightarrow \mathbf{1}$.

$$
I_{2}(x) \simeq x\left(-\frac{3}{2}-\ln x\right)+x^{2}\left(-\frac{16}{3}-4 \ln x\right)+\mathcal{O}\left(x^{3}\right),
$$

$I_{3}(x) \simeq x\left(-\frac{11}{6}-\ln x\right)+x^{2}\left(-\frac{89}{12}-5 \ln x\right)+\mathcal{O}\left(x^{3}\right)$,

and, thus, for $m_{\ell} \ll m_{S}$,

$$
\begin{aligned}
\Delta a_{\ell}^{(1)}= & \frac{1}{8 \pi^{2}} \frac{m_{\ell}^{2}}{m_{S}^{2}} \frac{m_{\ell}^{2}}{v^{2}}\left\{-\left[a_{\ell}^{S}\right]^{2}\left(\frac{7}{6}+\ln \left(\frac{m_{\ell}^{2}}{m_{S}^{2}}\right)\right)\right. \\
& \left.+\left[b_{\ell}^{S}\right]^{2}\left(\frac{11}{6}+\ln \left(\frac{m_{\ell}^{2}}{m_{S}^{2}}\right)\right)\right\} .
\end{aligned}
$$

Yukawa interactions (of charged scalars $C^{ \pm}$) of the form

$\mathcal{L}_{C \ell \nu}=-C^{-} \bar{\ell}\left(a_{\ell}^{C}+i b_{\ell}^{C} \gamma_{5}\right) \nu-C^{+} \bar{\nu}\left(a_{\ell}^{C *}+i b_{\ell}^{C *} \gamma_{5}\right) \ell$,

give one loop contributions to the anomalous magnetic moment of lepton $\ell$ of the form

$$
\Delta a_{\ell}^{(1)}=-\frac{1}{8 \pi^{2}} \sum_{C}\left\{\left|a_{\ell}^{C}\right|^{2}+\left|b_{\ell}^{C}\right|^{2}\right\} H\left(x_{\ell C}\right),
$$

where $x_{\ell C}=m_{\ell}^{2} / m_{C^{ \pm}}^{2}$, and

$$
\begin{aligned}
& H(x)=-\frac{1}{2}+\frac{1}{x}+\frac{1-x}{x^{2}} \ln (1-x), \\
& H(x) \simeq \frac{x}{6}+\frac{x^{2}}{12}+\mathcal{O}\left(x^{3}\right) \quad \text { for } x \ll 1 .
\end{aligned}
$$

\section{Two loop contributions}

In addition to Eq. (B1), Yukawa interactions of the form

$$
\mathcal{L}_{S \overline{f f}}=-\frac{m_{f}}{v} S \bar{f}\left(\alpha_{f}^{S}+i \beta_{f}^{S} \gamma_{5}\right) f
$$

give the following type of two loop Barr-Zee contributions to the anomalous magnetic moment of lepton $\ell$ : 


$$
\begin{aligned}
\Delta a_{\ell}^{(2)}= & -\frac{\alpha^{2}}{4 \pi^{2} s_{W}^{2}} \frac{m_{\ell}^{2}}{M_{W}^{2}} \\
& \times \sum_{f} \sum_{S} N_{c}^{f} Q_{f}^{2}\left\{a_{\ell}^{S} \alpha_{f}^{S} f\left(z_{f S}\right)-b_{\ell}^{S} \beta_{f}^{S} g\left(z_{f S}\right)\right\} .
\end{aligned}
$$

The sum over fermions $f$ corresponds to the different fermions appearing in the closed fermion loop (with $N_{c}^{f}$ the number of colours of $f$ and $Q_{f}$ its electric charge and $z_{f S}=m_{f}^{2} / m_{S}^{2}$, while the sum over scalars $S$ corresponds to the different neutral scalars connecting the closed fermion loop with the external lepton line, as Fig. 11 illustrates. The functions $f(z)$ and $g(z)$ (see the discussion in Sec. III) read:

$$
\begin{aligned}
& f(z)=\frac{z}{2} \int_{0}^{1} d x \frac{1-2 x(1-x)}{x(1-x)-z} \ln \left(\frac{x(1-x)}{z}\right), \\
& g(z)=\frac{z}{2} \int_{0}^{1} d x \frac{1}{x(1-x)-z} \ln \left(\frac{x(1-x)}{z}\right) .
\end{aligned}
$$

For other 2 loop contributions see [59].
[1] R. H. Parker, C. Yu, W. Zhong, B. Estey, and H. Muller, Science 360, 191 (2018).

[2] H. Davoudiasl and W. J. Marciano, Phys. Rev. D 98, 075011 (2018).

[3] T. Aoyama, M. Hayakawa, T. Kinoshita, and M. Nio, Phys. Rev. Lett. 109, 111807 (2012).

[4] T. Aoyama, M. Hayakawa, T. Kinoshita, and M. Nio, Phys. Rev. Lett. 109, 111808 (2012).

[5] S. Laporta, Phys. Lett. B 772, 232 (2017).

[6] T. Aoyama, T. Kinoshita, and M. Nio, Phys. Rev. D 97, 036001 (2018).

[7] S. Volkov, Phys. Rev. D 100, 096004 (2019).

[8] H. Terazawa, Nonlin. Phenom. Complex Syst. 21, 268 (2018), https://inspirehep.net/literature/1707568.

[9] G. W. Bennett et al. (Muon g-2 Collaboration), Phys. Rev. D 73, 072003 (2006).

[10] F. Jegerlehner and A. Nyffeler, Phys. Rep. 477, 1 (2009).

[11] M. Davier, A. Hoecker, B. Malaescu, and Z. Zhang, Eur. Phys. J. C 71, 1515 (2011); 72, 1874(E) (2012).

[12] M. Davier, A. Hoecker, B. Malaescu, and Z. Zhang, Eur. Phys. J. C 80, 241 (2020); 80, 410(E) (2020).

[13] T. Blum, P. Boyle, V. Gülpers, T. Izubuchi, L. Jin, C. Jung, A. Jüttner, C. Lehner, A. Portelli, and J. Tsang (RBC, UKQCD Collaborations), Phys. Rev. Lett. 121, 022003 (2018).

[14] T. Aoyama et al., arXiv:2006.04822.

[15] P. Roig and P. Sanchez-Puertas, Phys. Rev. D 101, 074019 (2020).

[16] G. F. Giudice, P. Paradisi, and M. Passera, J. High Energy Phys. 11 (2012) 113.

[17] A. Crivellin, M. Hoferichter, and P. Schmidt-Wellenburg, Phys. Rev. D 98, 113002 (2018).

[18] J. Liu, C. E. Wagner, and X.-P. Wang, J. High Energy Phys. 03 (2019) 008.

[19] X.-F. Han, T. Li, L. Wang, and Y. Zhang, Phys. Rev. D 99, 095034 (2019).

[20] M. Endo and W. Yin, J. High Energy Phys. 08 (2019) 122.

[21] M. Bauer, M. Neubert, S. Renner, M. Schnubel, and A. Thamm, Phys. Rev. Lett. 124, 211803 (2020).

[22] M. Badziak and K. Sakurai, J. High Energy Phys. 10 (2019) 024.
[23] G. Hiller, C. Hormigos-Feliu, D. F. Litim, and T. Steudtner, arXiv:1910.14062.

[24] A. C. Hernández, Y. H. Velásquez, S. Kovalenko, H. Long, N. A. Pérez-Julve, and V. Vien, arXiv:2002.07347.

[25] N. Haba, Y. Shimizu, and T. Yamada, arXiv:2002.10230.

[26] I. Bigaran and R. R. Volkas, arXiv:2002.12544.

[27] L. Calibbi, M. López-Ibáñez, A. Melis, and O. Vives, J. High Energy Phys. 06 (2020) 087.

[28] C.-H. Chen and T. Nomura, arXiv:2003.07638.

[29] S. Jana, P. K. Vishnu, and S. Saad, Phys. Rev. D 101, 115037 (2020).

[30] C. Hati, J. Kriewald, J. Orloff, and A. Teixeira, J. High Energy Phys. 07 (2020) 235.

[31] B. Dutta, S. Ghosh, and T. Li, arXiv:2006.01319.

[32] D. Sabatta, A. S. Cornell, A. Goyal, M. Kumar, B. Mellado, and X. Ruan, Chin. Phys. C 44, 063103 (2020).

[33] T. Lee, Phys. Rev. D 8, 1226 (1973).

[34] G. Branco, P. Ferreira, L. Lavoura, M. Rebelo, M. Sher, and J. P. Silva, Phys. Rep. 516, 1 (2012).

[35] I. P. Ivanov, Prog. Part. Nucl. Phys. 95, 160 (2017).

[36] S. L. Glashow and S. Weinberg, Phys. Rev. D 15, 1958 (1977).

[37] H. E. Haber, G. L. Kane, and T. Sterling, Nucl. Phys. B161, 493 (1979).

[38] V. D. Barger, J. L. Hewett, and R. J. N. Phillips, Phys. Rev. D 41, 3421 (1990).

[39] A. Pich and P. Tuzon, Phys. Rev. D 80, 091702 (2009).

[40] P. Ferreira, L. Lavoura, and J. P. Silva, Phys. Lett. B 688, 341 (2010).

[41] F. J. Botella, G. C. Branco, A. M. Coutinho, M. N. Rebelo, and J. I. Silva-Marcos, Eur. Phys. J. C 75, 286 (2015).

[42] C. B. Braeuninger, A. Ibarra, and C. Simonetto, Phys. Lett. B 692, 189 (2010).

[43] M. Jung, A. Pich, and P. Tuzon, J. High Energy Phys. 11 (2010) 003.

[44] A. Peñuelas and A. Pich, J. High Energy Phys. 12 (2017) 084.

[45] F. J. Botella, F. Cornet-Gomez, and M. Nebot, Phys. Rev. D 98, 035046 (2018).

[46] W. Rodejohann and U. Saldaña-Salazar, J. High Energy Phys. 07 (2019) 036. 
[47] H. Georgi and D. V. Nanopoulos, Phys. Lett. 82B, 95 (1979).

[48] J. F. Donoghue and L. F. Li, Phys. Rev. D 19, 945 (1979).

[49] F. J. Botella and J. P. Silva, Phys. Rev. D 51, 3870 (1995).

[50] M. Nebot and J. P. Silva, Phys. Rev. D 92, 085010 (2015).

[51] V. Andreev et al. (ACME Collaboration), Nature (London) 562, 355 (2018).

[52] T. Han, S. K. Kang, and J. Sayre, J. High Energy Phys. 02 (2016) 097.

[53] J. P. Leveille, Nucl. Phys. B137, 63 (1978).

[54] S. M. Barr and A. Zee, Phys. Rev. Lett. 65, 21 (1990); 65, 2920(E) (1990).

[55] D. Chang, W.-Y. Keung, and T. C. Yuan, Phys. Rev. D 43, R14 (1991).

[56] K.-M. Cheung, C.-H. Chou, and O. C. W. Kong, Phys. Rev. D 64, 111301 (2001).

[57] K. Cheung, O. C. W. Kong, and J. S. Lee, J. High Energy Phys. 06 (2009) 020.

[58] V. Ilisie, J. High Energy Phys. 04 (2015) 077.

[59] A. Cherchiglia, P. Kneschke, D. Stöckinger, and H. Stöckinger-Kim, J. High Energy Phys. 01 (2017) 007.

[60] I. P. Ivanov and J. P. Silva, Phys. Rev. D 92, 055017 (2015).

[61] S. Kanemura, T. Kubota, and E. Takasugi, Phys. Lett. B 313, 155 (1993).

[62] A. G. Akeroyd, A. Arhrib, and E.-M. Naimi, Phys. Lett. B 490, 119 (2000).

[63] I. F. Ginzburg and I. P. Ivanov, Phys. Rev. D 72, 115010 (2005).

[64] J. Horejsi and M. Kladiva, Eur. Phys. J. C 46, 81 (2006).

[65] S. Kanemura and K. Yagyu, Phys. Lett. B 751, 289 (2015).

[66] B. Grinstein, C. W. Murphy, and P. Uttayarat, J. High Energy Phys. 06 (2016) 070.

[67] M. Nebot, arXiv:1911.02266.

[68] W. Grimus, L. Lavoura, O. Ogreid, and P. Osland, Nucl. Phys. B801, 81 (2008).

[69] G. Aad et al. (ATLAS, CMS Collaboration), J. High Energy Phys. 08 (2016) 045.

[70] M. Aaboud et al. (ATLAS Collaboration), J. High Energy Phys. 12 (2017) 024.

[71] A. M. Sirunyan et al. (CMS Collaboration), Phys. Lett. B 780, 501 (2018).

[72] A. M. Sirunyan et al. (CMS Collaboration), Phys. Lett. B 779, 283 (2018).

[73] V. Khachatryan et al. (CMS Collaboration), Phys. Lett. B 744, 184 (2015).

[74] M. Aaboud et al. (ATLAS Collaboration), Phys. Rev. Lett. 119, 051802 (2017).

[75] N. Kauer and G. Passarino, J. High Energy Phys. 08 (2012) 116.

[76] G. Aad et al. (ATLAS Collaboration), Eur. Phys. J. C 75, 335 (2015).

[77] V. Khachatryan et al. (CMS Collaboration), J. High Energy Phys. 09 (2016) 051.

[78] F. J. Botella, G. C. Branco, M. Nebot, and M. N. Rebelo, Eur. Phys. J. C 76, 161 (2016).

[79] M. Nebot, F. J. Botella, and G. C. Branco, Eur. Phys. J. C 79, 711 (2019).

[80] Y. Kuno and Y. Okada, Rev. Mod. Phys. 73, 151 (2001).
[81] M. Tanabashi et al. (Particle Data Group Collaboration), Phys. Rev. D 98, 030001 (2018).

[82] V. Cirigliano and I. Rosell, Phys. Rev. Lett. 99, 231801 (2007).

[83] A. Pich, Prog. Part. Nucl. Phys. 75, 41 (2014).

[84] E. J. Chun and J. Kim, J. High Energy Phys. 07 (2016) 110.

[85] M. Misiak et al., Phys. Rev. Lett. 98, 022002 (2007).

[86] A. Crivellin, A. Kokulu, and C. Greub, Phys. Rev. D 87, 094031 (2013).

[87] F. Botella, G. Branco, A. Carmona, M. Nebot, L. Pedro, and M. Rebelo, J. High Energy Phys. 07 (2014) 078.

[88] S. Schael et al. (ALEPH Collaboration), Eur. Phys. J. C 49, 411 (2007).

[89] M. Aaboud et al. (ATLAS Collaboration), J. High Energy Phys. 10 (2017) 182.

[90] M. Aaboud et al. (ATLAS Collaboration), J. High Energy Phys. 07 (2019) 117.

[91] A. M. Sirunyan et al. (CMS Collaboration), Phys. Lett. B 798, 134992 (2019).

[92] M. Aaboud et al. (ATLAS Collaboration), J. High Energy Phys. 01 (2018) 055.

[93] V. Khachatryan et al. (CMS Collaboration), J. High Energy Phys. 02 (2017) 048.

[94] A. M. Sirunyan et al. (CMS Collaboration), J. High Energy Phys. 09 (2018) 007.

[95] M. Aaboud et al. (ATLAS Collaboration), Phys. Lett. B 759, 555 (2016).

[96] M. Aaboud et al. (ATLAS Collaboration), J. High Energy Phys. 11 (2018) 085.

[97] A. M. Sirunyan et al. (CMS Collaboration), J. High Energy Phys. 07 (2019) 142.

[98] A. M. Sirunyan et al. (CMS Collaboration), J. High Energy Phys. 01 (2020) 096.

[99] A. M. Sirunyan et al. (CMS Collaboration), J. High Energy Phys. 07 (2020) 126.

[100] D. de Florian et al. (LHC Higgs Cross Section Working Group Collaboration), arXiv:1610.07922.

[101] R. V. Harlander and W. B. Kilgore, Phys. Rev. Lett. 88, 201801 (2002).

[102] V. Ravindran, J. Smith, and W. L. van Neerven, Nucl. Phys. B665, 325 (2003).

[103] A. Pak, M. Rogal, and M. Steinhauser, J. High Energy Phys. 09 (2011) 088.

[104] R. V. Harlander and W. B. Kilgore, J. High Energy Phys. 10 (2002) 017.

[105] C. Anastasiou and K. Melnikov, Phys. Rev. D 67, 037501 (2003).

[106] T. Ahmed, M. Bonvini, M. C. Kumar, P. Mathews, N. Rana, V. Ravindran, and L. Rottoli, Eur. Phys. J. C 76, 663 (2016).

[107] M. Flechl, R. Klees, M. Kramer, M. Spira, and M. Ubiali, Phys. Rev. D 91, 075015 (2015).

[108] C. Degrande, M. Ubiali, M. Wiesemann, and M. Zaro, J. High Energy Phys. 10 (2015) 145.

[109] J. Alwall, R. Frederix, S. Frixione, V. Hirschi, F. Maltoni, O. Mattelaer, H. S. Shao, T. Stelzer, P. Torrielli, and M. Zaro, J. High Energy Phys. 07 (2014) 079.

[110] A. Alloul, N. D. Christensen, C. Degrande, C. Duhr, and B. Fuks, Comput. Phys. Commun. 185, 2250 (2014). 
[111] C. Degrande, Comput. Phys. Commun. 197, 239 (2015).

[112] C. Degrande, Proc. Sci., Charged2014 (2015) 024 [arXiv: 1412.6955].
[113] J. F. Gunion and H. E. Haber, Phys. Rev. D 67, 075019 (2003).

[114] F. Faro, J. C. Romao, and J. P. Silva, Eur. Phys. J. C 80, 635 (2020). 